\title{
Deformation of a Kac Algebra by an Abelian Subgroup
}

\author{
Michel Enock ${ }^{1}$, Leonid Vănerman ${ }^{2}$ \\ ${ }^{1}$ Unité de Recherche Associée au CNRS 747, Case 191, Université Pierre et Marie Curie, F-75252 \\ Paris Cedex 05, France \\ 2 International Solomon University, Zabolotny Street, 38, Apt. 61, 252187 Kiev, Ukraine
}

Received: 1 June 1995/Accepted: 27 September 1995

Dedicated to the memory of George Кас (Георай Исаакович Кач)

\begin{abstract}
Some examples of quantum groups in literature arise as deformations of a locally compact group by a "dual" 2-cocycle. We make this construction in the framework of Kac algebras; we show that these deformations are still Kac algebras; using this construction, we give new quantizations of the Heisenberg group. From this point of view, we analyse the dimension 8 non-trivial example of Kac and Paljutkin, and give a new example of non-trivial dimension 12 semi-simple $*$-Hopf algebras (a dimension $12 \mathrm{Kac}$ algebra).
\end{abstract}

\section{Introduction}

1.1. The first attempts to define group-like structures (what is now called "quantum groups") with the help of operator algebras were made to clarify the duality of locally compact (non-abelian) groups, this group-like structure then to be put on the "dual" of the locally compact group. At the algebraic level, the notion of Hopf algebras is commonly used to deal with discrete groups and their dual objects at the same time, and therefore, to reach the same aim for general locally compact groups, because of the analysis of infinite dimensional unitary representations, it was more or less natural to mix up operator algebras and the algebraic framework of Hopf algebras. This was made in the 60's by G.I. Kac, who constructed the "ring-groups," a category which contained both unimodular groups and their duals; the non-unimodular case, i.e. to construct a wider category which contains both locally compact groups and their duals, was done in the 70's, after the works of M. Takesaki, independently by G.I. Kac and the second author, and by J.-M. Schwartz and the first author, who named "Kac algebras" this wider category, in order to emphasize Kac's 1961 basic work. On that theory, we refer to [ES2].

1.2. Unfortunately, this theory suffered for a long time from a serious lack of non-trivial examples (see [KP1] and [KP2] for the first non-trivial examples); on

Research of the second author was supported in part by the Ukrainian Foundation for Fundamental Studies and by the International Science Foundation. 
the contrary, from the study of the quantum inverse scattering method, a "quantization" procedure was made by V.G. Drinfeld [D] and others in order to obtain non-commutative and non-co-commutative Hopf algebras by deformations of the enveloping algebras of semi-simple Lie algebras. Independently, S.L. Woronowicz [W1] constructed a $\mathbb{C}^{*}$-Hopf-algebra which was a deformation of the algebra of continuous functions on the compact Lie group $S U(2)$; this last object, which was essentially the same as Drinfeld's construction, satisfied weaker properties than the axioms of Kac algebras, and was the first example of what is now called a "compact quantum group." A satisfactory theory of compact quantum groups is now available ([W2]), and, by duality, the discrete case has been studied also ([PW,ER]).

1.3. Unless no precise definition of a "locally compact quantum group" is still given (see $[\mathrm{MN}]$ on that subject), many mathematicians are now involved in searching (and finding) concrete examples; historically, the first known examples were given in [KP1] and [KP2]. Many works appeared recently ([L, LR, R1, R2, V]); in particular, in ([L], Sects. 1 and 2), Landstad made a general construction of quantum groups, taking the von Neumann algebra $\mathscr{L}(G)$ generated by the left regular representation $\lambda_{G}$ of a locally compact group $G$, and deforming the canonical coproduct $\Gamma_{s}^{G}$ of $\mathscr{L}(G)$, which is defined, for all $g$ in $G$, by:

$$
\Gamma_{s}^{G}\left(\lambda_{G}(g)\right)=\lambda_{G}(g) \otimes \lambda_{G}(g)
$$

in order to obtain a new coproduct which can be written, for all $x$ in $\mathscr{L}(G)$ :

$$
\Gamma_{\Omega}(x)=\Omega \Gamma_{s}^{G}(x) \Omega^{*},
$$

where $\Omega$ is a "dual" 2-cocycle lifted from an abelian subgroup $H$ of $G$. The notion of triangular QUE-algebra described in ([D] Sect. 10) is very similar.

This procedure, deforming the coproduct of $\mathscr{L}(G)$, gives, on the predual $A(G)$ of $\mathscr{L}(G)$, a deformation of the product; it is the point of view developed by Rieffel in ([R1], Sect. 1) or ([R2], Sect. 2). This leads to the dual situation, where one takes an algebra of functions on $G$, deform the product on this algebra, and leaves untouched the canonical coproduct on this algebra. These two procedures are dual to each other, and therefore equivalent. For a generalisation of Rieffel's point of view, see [Wa].

1.4. The aim of this paper is to show that, on certain conditions, Landstad's deformations of an ordinary locally compact group are Kac algebras, to get generalizations of this construction for Kac algebras, and to show that the result of such a deformation is again a Kac algebra. One should notice that another construction of a deformation of a coproduct (or a multiplicative unitary), using a 2-cocycle, is to be found in ([BS], 8.24, 8.26).

1.5. The paper is organized as follows: in the second section, we define 2-cocycles and 2-pseudo-cocycles for a von Neumann algebra $M$ equipped with a coassociative coproduct $\Gamma$ (Hopf-von Neumann algebra), and, for any such object $\Omega$ the deformation $\Gamma_{\Omega}$ of the coproduct associated; if, more precisely, there is a co-involution $\kappa$, we give the definition of a 2-cocycle and a 2-pseudo-cocycle with respect to $(M, \Gamma, \kappa)$, and, for such an $\Omega$, we get then that $\left(M, \Gamma_{\Omega}, \kappa\right)$ remains a co-involutive Hopf-von Neumann algebra.

In the third section, we start with a Woronowicz algebra; it is a class of objects introduced in $[\mathrm{MN}]$, which contains Kac algebras, compact and discrete quantum 
groups. Then, the constructions obtained in Sect. 2 with 2-cocycles allows us to define a multiplicative unitary, which is the deformation of the multiplicative unitary associated to the Woronowicz algebra.

In Sect. 4, following Landstad, we consider 2-cocycles on Kac algebras given by lifting special 2-cocycles associated to an abelian group.

In Sect. 5 the main result of this paper is given: in the case of Kac algebras, when the 2-cocycle is of the type described in Sect. 4, and lies in the centralizer of the Haar weight, then the coinvolutive Hopf-von Neumann algebra obtained in Sect. 2 is another Kac algebra, whose multiplicative unitary is the deformed multiplicative unitary obtained in Sect. 3.

In Sect. 6, we give some examples; more precisely, we show that Landstad's construction of the deformation of a locally compact group arising from an abelian subgroup, is, by our result, a Kac algebra; this allows us to give new quantizations of the Heisenberg group. Moreover, we show that the dimension 8 example of the non-trivial Kac algebra given in the 60's by Kac and Paljutkin [KP2] is a deformation given by a pseudo-2-cocycle of a symmetric Kac algebra. Using the same techniques, we construct a new example of a dimension 12 non trivial Kac algebra.

1.6. As a final remark, we must notice that, in our construction, the co-involution remains undeformed. So, the examples [KP1] and [V] cannot be described in that way.

1.7. We are thankful to Eduard Vaysleb for references to the papers on finite dimensional Hopf algebras, and to Masaki Izumi for pointing out a mistake in a preliminary version of this work.

\section{Cocycles of Hopf-von Neumann Algebras}

2.1. Definition. A Hopf-von Neumann algebra is a von Neumann algebra $M$ with an injective morphism $\Gamma$ from $M$ to the von Neumann tensor product $M \otimes M$ such as:

$$
\begin{gathered}
\Gamma(1)=1, \\
(\Gamma \otimes i) \Gamma=(i \otimes \Gamma) \Gamma,
\end{gathered}
$$

where $i$ means the identity of $M$ (cf. [ES2], 1.2.1).

2.2. Definition. Let $(M, \Gamma)$ be a Hopf-von Neumann algebra as defined in 2.1 ; for all unitary $u$ in $M$, we define $\partial_{1} u$ in $M \otimes M$ by

$$
\partial_{1} u=\left(u^{*} \otimes u^{*}\right) \Gamma(u)
$$

and, for all unitary $\Omega$ in $M \otimes M$, we define $\partial_{2} \Omega$ in $M \otimes M \otimes M$ by

$$
\partial_{2} \Omega=(i \otimes \Gamma)\left(\Omega^{*}\right)\left(1 \otimes \Omega^{*}\right)(\Omega \otimes 1)(\Gamma \otimes i)(\Omega) .
$$

2.3. Definition. Let $(M, \Gamma)$ be a Hopf-von Neumann algebra as defined in 2.1 ; we shall call a 2-cocycle of $(M, \Gamma)$ a unitary $\Omega$ in $M \otimes M$ such that $\partial_{2} \Omega=1$, or, equivalently:

$$
(\Omega \otimes 1)(\Gamma \otimes i)(\Omega)=(1 \otimes \Omega)(i \otimes \Gamma)(\Omega) .
$$


We shall call a 2-pseudo-cocycle of $(M, \Gamma)$ a unitary $\Omega$ in $M \otimes M$ such that $\partial_{2} \Omega$ belongs to $(\Gamma \otimes i) \Gamma(M)^{\prime}$.

2.4. Examples. Let $G$ be a locally compact group, and let $L^{\infty}(G)$ be the abelian von Neumann algebra of the (classes of) essentially bounded measurable (with respect to the left Haar measure $d s$ ) complex-valued functions on $G$. Then, it is well known that the von Neumann tensor product $L^{\infty}(G) \otimes L^{\infty}(G)$ can be identified with $L^{\infty}(G \times G)$; for every $f$ in $L^{\infty}(G)$, let us define a two variable function $\Gamma_{a}^{G}(f)$ by, for all $s, t$ in $G$ :

$$
\Gamma_{a}^{G}(f)(s, t)=f(s t) .
$$

Then $\left(L^{\infty}(G), \Gamma_{a}^{G}\right)$ is a Hopf-von Neumann algebra ([ES2], 1.2.9), and a 2-cocycle of $\left(L^{\infty}(G), \Gamma_{a}^{G}\right)$ is a (class of) measurable function $\omega$ from $G \times G$ to $\mathbb{T}$, such that, for almost all $s_{1}, s_{2}, s_{3}$ in $G$ :

$$
\omega\left(s_{1}, s_{2}\right) \omega\left(s_{1} s_{2}, s_{3}\right)=\omega\left(s_{2}, s_{3}\right) \omega\left(s_{1}, s_{2} s_{3}\right) .
$$

Such a function will be called a 2-cocycle on $G$.

As $L^{\infty}(G)$ is abelian, it is clear that any measurable function $\omega$ from $G \times G$ to $\mathbb{T}$ is a 2-pseudo-cocycle of $\left(L^{\infty}(G), \Gamma_{a}^{G}\right)$.

2.5. Proposition. Let $(M, \Gamma)$ be a Hopf-von Neumann algebra, and $\Omega$ a 2-pseudococycle of $(M, \Gamma)$; then, let us put, for all $x$ in $M$ :

$$
\Gamma_{\Omega}(x)=\Omega \Gamma(x) \Omega^{*} .
$$

Then, $\left(M, \Gamma_{\Omega}\right)$ is a Hopf-von Neumann algebra, and we shall say that $\left(M, \Gamma_{\Omega}\right)$ (or $\left.\Gamma_{\Omega}\right)$ is deformed from $(M, \Gamma)$ (or from $\Gamma$ ) by $\Omega$.

Proof. This is clear by the following calculation:

$$
\begin{aligned}
\left(\Gamma_{\Omega} \otimes i\right) \Gamma_{\Omega}(x) & =(\Omega \otimes 1)(\Gamma \otimes i)\left(\Omega \Gamma(x) \Omega^{*}\right)\left(\Omega^{*} \otimes 1\right) \\
& =(\Omega \otimes 1)(\Gamma \otimes i)(\Omega)(\Gamma \otimes i)(\Gamma(x))(\Gamma \otimes i)\left(\Omega^{*}\right)\left(\Omega^{*} \otimes 1\right) \\
& =(1 \otimes \Omega)(i \otimes \Gamma)(\Omega) \partial_{2} \Omega(i \otimes \Gamma)(\Gamma(x))\left(\partial_{2} \Omega\right)^{*}(i \otimes \Gamma)\left(\Omega^{*}\right)\left(1 \otimes \Omega^{*}\right) \\
& =(1 \otimes \Omega)(i \otimes \Gamma)\left(\Gamma_{\Omega}(x)\right)\left(1 \otimes \Omega^{*}\right) \\
& =\left(i \otimes \Gamma_{\Omega}\right)\left(\Gamma_{\Omega}(x)\right) .
\end{aligned}
$$

2.6. Remark. (i) For any unitary $u$ of $M$, it is easy to check that $\partial_{1} u$ is a 2-cocycle for $(M, \Gamma)$.

(ii) Let $\varsigma$ be the flip of $M \otimes M$; it is clear that $(M, \varsigma \Gamma)$ is another Hopfvon Neumann algebra; if $\Omega$ is a 2 -(pseudo-)cocycle for $(M, \Gamma)$, then $\varsigma \Omega$ is a 2-(pseudo-)cocycle for $(M, \varsigma \Gamma)$.

(iii) If $\Omega$ is a 2 -(pseudo-)cocycle for $(M, \Gamma)$, then $\Omega^{*}$ is a 2 -(pseudo-)cocycle for $\left(M, \Gamma_{\Omega}\right)$.

(iv) Let $V$ be a multiplicative unitary in the sense of [BS], i.e. a unitary on the Hilbert tensor product $\mathfrak{H} \otimes \mathfrak{H}$, such that $V$ satisfies the pentagonal equation:

$$
V_{12} V_{13} V_{23}=V_{23} V_{12} \text {, }
$$

then, if $M$ is the von Neumann algebra generated by all the operators $(i \otimes \omega)(V)$, where $\omega$ belongs to the predual $\mathscr{L}(\mathfrak{S})_{*}$, the application $x \rightarrow V(x \otimes 1) V^{*}$ defines a 
coproduct $\Gamma$ on $M$, and a 2-cocycle for $(M, \Gamma)$ is a unitary $\Omega$ in $M \otimes M$ satisfying:

$$
\Omega_{12} V_{12} \Omega_{13} V_{12}^{*}=\Omega_{23} V_{23} \Omega_{12} V_{23}^{*} .
$$

A pseudo-2-cocycle is a unitary $\Omega$ in $M \otimes M$ such that $V_{23} \Omega_{12}^{*} V_{23}^{*} \Omega_{23}^{*} \Omega_{12} V_{12} \Omega_{13} V_{12}^{*}$ belongs to the commutant of $V_{12} V_{13} M_{1} V_{13}^{*} V_{12}^{*}$; using the pentagonal equation, we get that, equivalently, $\Omega$ must be such that $V_{12}^{*} \Omega_{12}^{*} V_{23}^{*} \Omega_{23}^{*} \Omega_{12} V_{12} \Omega_{13} V_{13}$ belongs to $Z(M) \otimes \mathscr{L}(\mathfrak{H} \otimes \mathfrak{H})$, where $Z(M)$ is the center of $M$.

(v) Let $\left(M_{1}, \Gamma_{1}\right)$ and $\left(M_{2}, \Gamma_{2}\right)$ be two Hopf-von Neumann algebras; let $\alpha$ be a $*$-homomorphism from $M_{1}$ to $M_{2}$ such that $\alpha(1)=1$ and $\Gamma_{2} \circ \alpha=(\alpha \otimes \alpha) \circ \Gamma_{1}$; then, if $\Omega$ is a 2-cocycle for $\left(M_{1}, \Gamma_{1}\right)$, then $(\alpha \otimes \alpha)(\Omega)$ is a 2-cocycle for $\left(M_{2}, \Gamma_{2}\right)$.

2.7. Definition. ([ES2], 1.2.5) A triple $(M, \Gamma, \kappa)$ is called a co-involutive Hopf-von Neumann algebra if:

(i) $(M, \Gamma)$ is a Hopf-von Neumann algebra as defined in 2.1,

(ii) $\kappa$ is an involutive anti-automorphism of $M$, i.e. a linear mapping from $M$ to $M$ such that, for every $x, y$ of $M$ :

$$
\begin{gathered}
\kappa(x y)=\kappa(y) \kappa(x), \\
\kappa\left(x^{*}\right)=\kappa(x)^{*}, \\
\kappa(\kappa(x))=x,
\end{gathered}
$$

such that we have

$$
\Gamma \circ \kappa=\varsigma \circ(\kappa \otimes \kappa) \circ \Gamma .
$$

2.8. Definition. Let $(M, \Gamma, \kappa)$ be a co-involutive Hopf-von Neumann algebra, $\Omega$ be a 2-pseudo-cocycle for $(M, \Gamma)$. We shall say that $\Omega$ is a 2-pseudo-cocycle for $(M, \Gamma, \kappa)$ if we have:

$$
(\kappa \otimes \kappa)(\Omega)=\Omega=\varsigma \Omega^{*} .
$$

We define the same way the 2-cocycles for $(M, \Gamma, \kappa)$.

2.9. Remark. (i) Let $G$ be a locally compact group, and let us define, for all $f$ in $L^{\infty}(G), \kappa_{a}(f)$ by the equality, for all $s$ in $G$ :

$$
\kappa_{a}^{G}(f)(s)=f\left(s^{-1}\right) .
$$

Then, $\left(L^{\infty}(G), \Gamma_{a}^{G}, \kappa_{a}^{G}\right)$ is a co-involutive Hopf-von Neumann algebra ([ES2] 1.2.9); let $\omega$ be a 2-cocycle on $G$, as defined in 2.4 ; it is a 2-cocycle for $\left(L^{\infty}(G), \Gamma_{a}^{G}, \kappa_{a}^{G}\right)$ if we have the equalities, for almost all $s, t$ in $G$ :

$$
\omega\left(s^{-1}, t^{-1}\right)=\bar{\omega}(t, s)=\omega(s, t) .
$$

We shall say then that $\omega$ is an involutive cocycle on $G$.

(ii) let $(M, \Gamma, \kappa)$ be a co-involutive Hopf-von Neumann algebra, and $\Omega$ be a 2-(pseudo-)cocycle for $(M, \Gamma)$; then it is straightforward to check that $(\kappa \otimes \kappa)\left(\Omega^{*}\right)$ is a 2-(pseudo-)cocycle for $(M, \varsigma \Gamma)$, and, therefore, using 2.6, that $\varsigma(\kappa \otimes \kappa)\left(\Omega^{*}\right)$ is a 2-(pseudo-)cocycle for $(M, \Gamma)$.

(iii) let $\left(M_{1}, \Gamma_{1}, \kappa_{1}\right)$ and $\left(M_{2}, \Gamma_{2}, \kappa_{2}\right)$ be two co-involutive Hopf-von Neumann algebras and let $\alpha$ be a morphism of co-involutive Hopf-von Neumann algebras, i.e. $\alpha$ is a $*$-homomorphism from $M_{1}$ to $M_{2}$ such that $\alpha(1)=1, \Gamma_{2} \circ \alpha=(\alpha \otimes \alpha) \circ \Gamma_{1}$ 
and $\kappa_{2} \circ \alpha=\alpha \circ \kappa_{1}$; then, if $\Omega$ is a 2-cocycle for $\left(M_{1}, \Gamma_{1}, \kappa_{1}\right)$, then $\alpha(\Omega)$ is a 2 cocycle for $\left(M_{2}, \Gamma_{2}, \kappa_{2}\right)$.

2.10. Proposition. Let $(M, \Gamma, \kappa)$ be a co-involutive Hopf-von Neumann algebra, $\Omega$ be a 2-pseudo-cocycle of $(M, \Gamma, \kappa)$; then $\left(M, \Gamma_{\Omega}, \kappa\right)$ is a co-involutive Hopf-von Neumann algebra.

Proof. Let $x$ be in $M$; we have, using 2.8:

$$
\begin{aligned}
\varsigma(\kappa \otimes \kappa) \Gamma_{\Omega}(x) & =\varsigma(\kappa \otimes \kappa)\left(\Omega \Gamma(x) \Omega^{*}\right) \\
& =\varsigma(\kappa \otimes \kappa)\left(\Omega^{*}\right) \varsigma(\kappa \otimes \kappa)(\Gamma(x)) \varsigma(\kappa \otimes \kappa)\left(\Omega^{*}\right) \\
& =\Omega \Gamma \circ \kappa(x) \Omega^{*}=\Gamma_{\Omega} \circ \kappa(x) .
\end{aligned}
$$

\section{Cocycles of Kac Algebras and Woronowicz Algebras}

3.1. Definition. A Kac algebra ([ES2] 2.2.5) is a quadruple $\mathbb{K}=(M, \Gamma, \kappa, \varphi)$ such that:

(i) $(M, \Gamma, \kappa)$ is a co-involutive Hopf-von Neumann algebra;

(ii) $\varphi$ is a semi-finite faithful normal weight on $M$ satisfying, for all $x$ in $M^{+}$, $y, z$ in the left ideal $\mathfrak{N}_{\varphi}$, $t$ in $\mathbb{R}$ :

$$
\begin{gathered}
(i \otimes \varphi) \Gamma(x)=\varphi(x) 1, \\
(i \otimes \varphi)\left(\left(1 \otimes y^{*}\right) \Gamma(z)\right)=\kappa\left((i \otimes \varphi)\left(\Gamma\left(y^{*}\right)(1 \otimes z)\right)\right), \\
\kappa \circ \sigma_{t}^{\varphi}=\sigma_{-t}^{\varphi} \circ \kappa .
\end{gathered}
$$

There is an equivalent $\mathbb{C}^{*}$-version of this theory [EV]; more precisely, it is possible to define $\mathbb{C}^{*}$-Kac algebras and to prove: algebra

-that any $\mathbb{C}^{*}$-Kac algebra can be imbedded canonically in a unique Kac

-that for any Kac algebra $(M, \Gamma, \kappa, \varphi)$, there is a unique $\mathbb{C}^{*}$-subalgebra $A$ of $M$ such that $A$, equipped with the restrictions of $\Gamma, \kappa$ and $\varphi$ is a $\mathbb{C}^{*}$-Kac algebra.

3.2. Examples. (i) Let $G$ be a locally compact group; the left Haar measure $d s$ defines a semi-finite faithful normal weight $\varphi_{a}^{G}$ on the abelian von Neumann algebra $L^{\infty}(G)$; it is then straightforward to verify ([ES2] 2.2.2) that, with the notations of $2.9,\left(L^{\infty}(G), \Gamma_{a}^{G}, \kappa_{a}^{G}, \varphi_{a}^{G}\right)$ is a Kac algebra. Any Kac algebra whose underlying von Neumann algebra is abelian is of that type ([ES2] 4.2.5). The associated $\mathbb{C}^{*}$-Kac algebra is the $\mathbb{C}^{*}$-algebra $C_{0}(G)$ of all continuous functions on $G$ going to 0 at the infinity, equipped with the restrictions of these coproduct, coinvolution and weight.

(ii) Let $\lambda_{G}$ be the left regular representation of $G$ on the Hilbert space $L^{2}(G)$, defined for all $s, t$ in $G, f$ in $L^{2}(G)$ by

$$
\left(\lambda_{G}(s) f\right)(t)=f\left(s^{-1} t\right) .
$$

Let $\mathscr{L}(G)$ be the von Neumann algebra generated by this representation; it is possible to define on $\mathscr{L}(G)$ a structure of Kac algebra ([ES2], 3.7.5) $\left(\mathscr{L}(G), \Gamma_{s}^{G}, \kappa_{s}^{G}, \varphi_{s}^{G}\right)$, such that, for any $s$ in $G$, any $f$ continuous function on $G$ with compact support, 
we have, where $e$ is the unit of $G$ :

$$
\begin{aligned}
\Gamma_{s}^{G}\left(\lambda_{G}(s)\right) & =\lambda_{G}(s) \otimes \lambda_{G}(s), \\
\kappa_{s}^{G}\left(\lambda_{G}(s)\right) & =\lambda_{G}\left(s^{-1}\right), \\
\varphi_{s}^{G}\left(\lambda_{G}(f)\right) & =f(e) .
\end{aligned}
$$

The modular group of $\varphi_{s}^{G}$ is given, for all $s$ in $G, t$ in $\mathbb{R}$, by:

$$
\sigma_{t}^{\varphi_{s}^{G}}\left(\lambda_{G}(s)\right)=\Delta_{G}(s)^{\mathrm{it}} \lambda_{G}(s),
$$

where $\Delta_{G}$ is the modulus of $G$. Any Kac algebra whose coproduct is symmetric (i.e. such that $\Gamma=\varsigma \circ \Gamma$ where $\varsigma$ is the flip) is of that type ([ES2], 4.2.4). The associated $\mathbb{C}^{*}$-Kac algebra is the $\mathbb{C}^{*}$-algebra $\mathbb{C}_{r}^{*}(G)$ generated by the left regular representation of $L^{1}(G)$, equipped with the restrictions of these coproduct, coinvolution and weight.

3.3. Remark. Let $\mathbb{K}=(M, \Gamma, \kappa, \varphi)$ be a Kac algebra; then, it is possible to define a unitary (the fundamental unitary of the Kac algebra) $W$ on the Hilbert space $H_{\varphi} \otimes H_{\varphi}$, such that, for all $x, y$ in the left ideal $\mathfrak{N}_{\varphi}$ :

$$
W\left(\Lambda_{\varphi}(x) \otimes \Lambda_{\varphi}(y)\right)=\Lambda_{\varphi \otimes \varphi}(\Gamma(y)(x \otimes 1)) .
$$

The adjoint $W^{*}$ is a multiplicative unitary in the sense of [BS]; the von Neumann algebra $M$ is equal to the von Neumann algebra generated by all $(i \otimes \omega)(W)$, for all $\omega$ in the predual $\mathscr{L}\left(H_{\varphi}\right)$, and we get, for all $x$ in $M$ :

$$
\Gamma(x)=W(1 \otimes x) W^{*} .
$$

Moreover, we have $W^{*}=(\hat{J} \otimes J) W(\hat{J} \otimes J)$, where $J$ is the canonical involutive isometry $J_{\varphi}$ constructed by the Tomita-Takesaki theory, and $\hat{J}$ is the canonical implementation on $H_{\varphi}$ of the anti-automorphism $\kappa$.

It is possible to associate another $\operatorname{Kac}$ algebra $(\hat{M}, \hat{\Gamma}, \hat{\kappa}, \hat{\varphi})$ (the dual Kac algebra), whose fundamental unitary is equal to $\sigma W^{*} \sigma$. The bidual Kac algebra is equal to the initial one, and the two examples above (3.2) are dual to each other. ([ES2], 2.4.2, 2.4.4, 2.6.2, 2.6.3, 2.7.6, 3.7.3, 3.7.5 and 4.1.1.)

3.4. Definition. A Woronowicz algebra $([\mathrm{MN}])$ is a family $\mathbb{W}=(M, \Gamma, \kappa, \tau, \varphi)$ such that:

(i) $(M, \Gamma, \kappa)$ is a co-involutive Hopf-von Neumann algebra;

(ii) $\tau_{t}$ is a one parameter group of *-automorphisms of $M$, such that, for all $t$ in $\mathbb{R}$, we have:

$$
\begin{gathered}
\Gamma \circ \tau_{t}=\left(\tau_{t} \otimes \tau_{t}\right) \circ \Gamma \\
\kappa \circ \tau_{t}=\tau_{t} \circ \kappa
\end{gathered}
$$

(iii) $\varphi$ is a semi-finite faithful normal weight on $M$ satisfying, for all $x$ in $M^{+}$, $y, z$ in the left ideal $\mathfrak{N}_{\varphi}, \omega$ in $M_{*}$ analytic with respect to the action of $\tau_{t}$ on $M_{*}$, $s, t$ in $\mathbb{R}$ :

$$
\begin{gathered}
(i \otimes \varphi) \Gamma(x)=\varphi(x) 1, \\
(\omega \otimes \varphi)\left(\left(1 \otimes y^{*}\right) \Gamma(z)\right)=\omega \circ \tau_{-i / 2} \circ \kappa\left((i \otimes \varphi)\left(\Gamma\left(y^{*}\right)(1 \otimes z)\right)\right), \\
\sigma_{t}^{\varphi} \circ \sigma_{s}^{\varphi \circ \kappa}=\sigma_{s}^{\varphi \circ \kappa} \circ \sigma_{t}^{\varphi} .
\end{gathered}
$$


As for Kac algebras, it is possible, with the same definition as in 3.3, to define a unitary $W$ (the Kac-Takesaki operator) which fulfills the same properties ([MN], 2.4 (i), (ii), (iii), 2.1.6 (i), (iii), 3.8 (i), 4.2).

There is a duality within this class of objects ([MN], Sect. 3), which generalizes the duality of Kac algebras, the dual Kac-Takesaki operator being equal to $\sigma W^{*} \sigma$ ([MN], 3.8).

3.5. Examples. (i) It is clear that any Kac algebra is a Woronowicz algebra.

(ii) Let $(A, d)$ be a compact quantum group, i.e. ([W2]) $A$ is a $\mathbb{C}^{*}$-algebra with unit, and $d$ is a unital *-homomorphism from $A$ to $A \otimes A$ such that $(d \otimes i) d$ $=(i \otimes d) d$, such that the set of all $(a \otimes 1) d b$, for all $a, b$ in $A$, is total in $A \otimes A$, and such that the set of all $(1 \otimes a) d b$, for all $a, b$ in $A$, is total in $A \otimes A$.

Then ([W2], 1.3), there exists a unique left-invariant state $\varphi$ on $A$, and, if we denote $\left(H_{\varphi}, \pi_{\varphi}, \xi_{\varphi}\right)$ the G.N.S. construction, we may put on $M=\pi_{\varphi}(A)^{\prime \prime}$ a structure of Woronowicz algebra (let us note that the vector state induced by $\xi_{\varphi}$ on $\pi_{\varphi}(A)$ is faithful). It is explicitly done in ([MN], Sect. 5) in a particular case, but it can be done in whole generality by using ([W2] 1.5 and [BS] 1.2.4).

(iii) In [ER] a notion of discrete quantum group has been defined, which appears ([ER],10) to be the dual notion of a compact quantum group; therefore, it is possible also to associate to each discrete quantum group a Woronowicz algebra.

3.6. Proposition. Let $\mathbb{K}=(M, \Gamma, \kappa, \varphi)$ be a Kac algebra (or $\mathbb{W}=(M, \Gamma, \kappa, \tau, \varphi)$ a Woronowicz algebra), $W$ its fundamental unitary, $\Omega$ a 2-pseudo-cocycle with respect to $(M, \Gamma)$. With the notations of 3.3 , let us put

$$
\tilde{\Omega}=(\hat{J} \otimes J) \Omega^{*}(\hat{J} \otimes J)
$$

and $W_{\Omega}=\Omega W \tilde{\Omega}$. Then, for all $x$ in $M$, we have:

$$
\Gamma_{\Omega}(x)=W_{\Omega}(1 \otimes x) W_{\Omega}^{*} .
$$

Proof. By definition of $J$, we get that $\tilde{\Omega}$ belongs to $M \otimes M^{\prime}$; therefore, the result is trivial.

3.7. Lemma. With the hypothesis of 3.6, let us suppose that $\Omega$ is a 2-cocycle for $(M, \Gamma, \kappa)$; we have then:

$$
\begin{aligned}
(\Gamma \otimes i)(\tilde{\Omega})\left(\Omega^{*} \otimes 1\right)= & (\sigma \otimes i)\left(1 \otimes W^{*}\right)(\sigma \otimes i)(1 \otimes \tilde{\Omega})(\sigma \otimes i) \\
& \times(1 \otimes W)(1 \otimes \tilde{\Omega})(\sigma \otimes i),
\end{aligned}
$$

where $\sigma$ means the flip on $H_{\varphi} \otimes H_{\varphi}$.

Proof. Let us write $j(x)=J x^{*} J$; we define this way an anti-isomorphism from $M$ to $M^{\prime}$, or from $M^{\prime}$ to $M$. We have then $\tilde{\Omega}=(\kappa \otimes j)(\Omega)$, and, therefore

$$
(\Gamma \otimes i)(\tilde{\Omega})=(\Gamma \otimes i)(\kappa \otimes j)(\Omega)=(\varsigma(\kappa \otimes \kappa) \otimes j)(\Gamma \otimes i)(\Omega) .
$$

Using 2.8, we then get:

$$
\begin{aligned}
(\Gamma \otimes i)(\tilde{\Omega})\left(\Omega^{*} \otimes 1\right) & =(\varsigma(\kappa \otimes \kappa) \otimes j)((\Omega \otimes 1)(\Gamma \otimes i)(\Omega)) \\
& =(\varsigma(\kappa \otimes \kappa) \otimes j)((1 \otimes \Omega)(i \otimes \Gamma)(\Omega)) \\
& =(\varsigma(\kappa \otimes \kappa) \otimes j)(i \otimes \Gamma)(\Omega)(\varsigma \otimes i)(1 \otimes \tilde{\Omega})
\end{aligned}
$$


which is equal, using the implementations of $j$ and $\kappa$, to

$$
\begin{aligned}
(\sigma & \otimes 1)(\hat{J} \otimes \hat{J} \otimes J)(1 \otimes W)(\sigma \otimes 1)\left(1 \otimes \Omega^{*}\right) \\
\quad & \times(\sigma \otimes 1)\left(1 \otimes W^{*}\right)(\hat{J} \otimes \hat{J} \otimes J)(1 \otimes \tilde{\Omega})(\sigma \otimes 1)
\end{aligned}
$$

and, using the property linking $W, W^{*}$ and $J$ and $\hat{J}$, is equal to

$$
\begin{aligned}
& (\sigma \otimes 1)\left(1 \otimes W^{*}\right)(\hat{J} \otimes \hat{J} \otimes J)(\sigma \otimes 1)\left(1 \otimes \Omega^{*}\right) \\
& \quad \times(\sigma \otimes 1)(\hat{J} \otimes \hat{J} \otimes J)(1 \otimes W)(1 \otimes \tilde{\Omega})(\sigma \otimes 1),
\end{aligned}
$$

which gives the result.

3.8. Proposition. With the hypothesis and notations of 3.7, we have:

$$
\left(\Gamma_{\Omega} \otimes i\right)\left(W_{\Omega}\right)=\left(W_{\Omega}\right)_{23}\left(W_{\Omega}\right)_{13}
$$

Proof. Using the definition, we get:

$$
\begin{aligned}
\left(\Gamma_{\Omega} \otimes i\right)\left(W_{\Omega}\right) & =(\Omega \otimes 1)(\Gamma \otimes i)(\Omega)(\Gamma \otimes i)(W)(\Gamma \otimes i)(\tilde{\Omega})\left(\Omega^{*} \otimes 1\right) \\
& =(1 \otimes \Omega)(i \otimes \Gamma)(\Omega)(\Gamma \otimes i)(W)(\Gamma \otimes i)(\tilde{\Omega})\left(\Omega^{*} \otimes 1\right)
\end{aligned}
$$

Using then the fact that $(\Gamma \otimes i)(W)=(1 \otimes W)(\sigma \otimes 1)(1 \otimes W)(\sigma \otimes 1)$, we get, using 3.7 that $(\Gamma \otimes i)(W)(\Gamma \otimes i)(\tilde{\Omega})\left(\Omega^{*} \otimes 1\right)$ is equal to

$$
(1 \otimes W)(1 \otimes \tilde{\Omega})(\sigma \otimes i)(1 \otimes W)(1 \otimes \tilde{\Omega})(\sigma \otimes i) .
$$

As $(i \otimes \Gamma)(\Omega)=(1 \otimes W)(\sigma \otimes 1)(1 \otimes \Omega)(\sigma \otimes 1)\left(1 \otimes W^{*}\right)$, we then get that

$$
(i \otimes \Gamma)(\Omega)(\Gamma \otimes i)(W)(\Gamma \otimes i)(\tilde{\Omega})\left(\Omega^{*} \otimes 1\right)\left(1 \otimes W^{*}\right)
$$

is equal to

$(1 \otimes W)(\sigma \otimes 1)(1 \otimes \Omega)(\sigma \otimes 1)(1 \otimes \tilde{\Omega})(\sigma \otimes i)(1 \otimes W)(1 \otimes \tilde{\Omega})(\sigma \otimes i)$ and, as $(\sigma \otimes 1)(1 \otimes \Omega)(\sigma \otimes 1)$ and $(1 \otimes \tilde{\Omega})$ commute, we finally get:

$$
\left(\Gamma_{\Omega} \otimes i\right)\left(W_{\Omega}\right)=\left(1 \otimes W_{\Omega}\right)(\sigma \otimes 1)\left(1 \otimes W_{\Omega}\right)(\sigma \otimes 1),
$$

which is the result.

3.9. Theorem. Let $\mathbb{K}=(M, \Gamma, \kappa, \varphi)$ be a Kac algebra (or $\mathbb{W}=(M, \Gamma, \kappa, \tau, \varphi)$ a Woronowicz algebra), $W$ its fundamental unitary, $\Omega$ a 2-cocycle with respect to $(M, \Gamma, \kappa)$. Let us put $\tilde{\Omega}=(\hat{J} \otimes J) \Omega^{*}(\hat{J} \otimes J)$, where $J$ is the canonical involutive isometry $J_{\varphi}$ constructed by the Tomita-Takesaki theory, and $\hat{J}$ is the canonical implementation on $H_{\varphi}$ of the anti-automorphism $\kappa$, and $W_{\Omega}=\Omega W \tilde{\Omega}$; then we get:

(i) $W_{\Omega}^{*}$ is a multiplicative unitary,

(ii) $W_{\Omega}^{*}=(\hat{J} \otimes J) W_{\Omega}(\hat{J} \otimes J)$. 
Proof. By 3.6 and 3.8, we get (i); by 3.3 and the definition of $\tilde{\Omega}$, (ii) is trivial.

\section{Abelian Cocycles}

4.1. Proposition. Let $K$ be an abelian locally compact group (with an additive notation), $\widehat{K}$ its Pontrjagin dual, and let $\pi$ be a continuous homomorphism from $K$ to $\widehat{K}$ such that, for all $s$ in $K$, we have:

$$
\langle\pi(s), s\rangle=1 .
$$

Then the function $\omega(s, t)=\langle\pi(s), t\rangle$ is an involutive cocycle on $K$; moreover, it satisfies, for all $s, t$ in $K, \omega(s, s t)=\omega(s, t)$.

Proof. Starting from $\langle\pi(s t), s t\rangle=1$ for all $s, t$ in $K$, we get:

$$
\langle\pi(t), s\rangle=\langle\pi(s t), s\rangle=\overline{\langle\pi(s t), t\rangle}=\overline{\langle\pi(s), t\rangle} .
$$

The rest is just straightforward calculations.

4.2. Example. Let $K_{1}, K_{2}$ be two abelian locally compact groups, $\widehat{K_{1}}, \widehat{K_{2}}$ their Pontjagin's duals; let $\pi_{1}$ be a continuous homomorphism from $K_{1}$ to $\widehat{K_{2}}$ and $\widehat{\pi_{1}}$ the dual homomorphism from $K_{2}$ to $\widehat{K_{1}}$. Then the homomorphism $\pi$ from $K=K_{1} \times K_{2}$ to $\widehat{K}=\widehat{K_{1}} \times \widehat{K_{2}}$ defined, for all $s$ in $K_{1}, t$ in $K_{2}$ by:

$$
\pi(s, t)=\left(\widehat{\pi}_{1}(t),-\pi_{1}(s)\right)
$$

satisfies the conditions of 4.1 .

4.3. Lemma. Let $(M, \Gamma, \kappa, \varphi)$ be a Kac algebra (or $\mathbb{W}=(M, \Gamma, \kappa, \tau, \varphi)$ a Woronowicz algebra), $W$ its fundamental unitary, $K$ an abelian locally compact group, $\alpha$ a co-involutive Hopf-von Neumann morphism from $\left(L^{\infty}(K), \Gamma_{a}^{K}, \kappa_{a}^{K}\right)$ to $(M, \Gamma, \kappa)$, $\omega$ an involutive cocycle on $K$ as in 4.1 , and let $\Omega=(\alpha \otimes \alpha)(\omega)$. Let $a_{i}$, $b_{l}$ in $L^{\infty}(K)$ such that $\Sigma_{i}\left(a_{i} \otimes b_{i}\right) \rightarrow_{i} \omega$ strongly, and of norm less or equal to 1 ; then:

(i) $\Sigma_{i}\left(a_{i} \otimes 1\right) \Gamma_{a}\left(b_{i}\right)$ is weakly converging to $\omega$.

(ii) $\sum_{i}\left(\alpha\left(a_{i}\right)^{*} \otimes J \alpha\left(b_{i}\right) J\right)$ is weakly converging to $\tilde{\Omega}$.

Proof. The finite sums $\Sigma_{l}\left(a_{i} \otimes 1\right) \Gamma_{a}\left(b_{l}\right)$ are converging to the function $(s, t) \rightarrow$ $\omega(s, s t)$, i.e. to $\omega$, which is (i).

We can remark, also, because of the particular form of $\omega$, that $\left(\kappa_{a} \otimes i\right)(\omega)=\omega^{*}$. The application $x \rightarrow J \alpha\left(x^{*}\right) J$ from $L^{\infty}(K)$ to $M$ is a homomorphism, and, therefore, all the finite sums $\Sigma_{i}\left(\alpha\left(a_{i}\right)^{*} \otimes J \alpha\left(b_{i}\right) J\right)$ are of norm less or equal to 1 . Let $\xi, \xi^{\prime}$, $\eta, \eta^{\prime}$ in $H_{\varphi}$; we then get:

$$
\begin{aligned}
\left(\left(\sum_{i} \alpha\left(a_{i}\right)^{*} \otimes J \alpha\left(b_{i}\right) J\right)\left(\xi \otimes \xi^{\prime}\right) \mid \eta \otimes \eta^{\prime}\right) & =\sum_{i}\left(\alpha\left(a_{i}\right)^{*} \xi \mid \eta\right)\left(J \alpha\left(b_{i}\right) J \xi^{\prime} \mid \eta^{\prime}\right) \\
& =\overline{\left(\Sigma_{i}\left(\alpha\left(a_{i}\right) \otimes \alpha\left(b_{i}\right)\right)\left(\eta \otimes J \xi^{\prime}\right) \mid \xi \otimes J \eta^{\prime}\right)}
\end{aligned},
$$


which is converging to:

$$
\overline{\left(\Omega\left(\eta \otimes J \xi^{\prime}\right) \mid \xi \otimes J \eta^{\prime}\right)}=\left(\Omega^{*}\left(\xi \otimes J \eta^{\prime}\right) \mid \eta \otimes J \xi^{\prime}\right)=\left(\left(\omega_{\xi, \eta} \otimes i\right)\left(\Omega^{*}\right) J \eta^{\prime} \mid J \xi^{\prime}\right)
$$

and, using the remark above, we get that it is equal to:

$$
\begin{aligned}
& \left.\left(\left(\omega_{\xi, \eta} \circ \alpha \otimes \alpha\right)\left(\kappa_{a} \otimes i\right)(\omega) J \eta^{\prime} \mid J \xi^{\prime}\right)=\left(\omega_{\xi, \eta} \circ \kappa \otimes i\right)(\Omega) J \eta^{\prime} \mid J \xi^{\prime}\right) \\
& \quad=\left(\left(\omega_{\hat{J} \eta, \hat{J} \xi} \otimes i\right)(\Omega) J \eta^{\prime} \mid J \xi^{\prime}\right)=\left(\Omega\left(\hat{J} \eta \otimes J \eta^{\prime}\right) \mid \hat{J} \xi \otimes J \xi^{\prime}\right) \\
& \quad=\left(\tilde{\Omega}\left(\xi \otimes \xi^{\prime}\right) \mid \eta \otimes \eta^{\prime}\right)
\end{aligned}
$$

and, by linearity and continuity, we get (ii).

4.4. Remark. As $\omega$ is multiplicative in the first variable, we get:

$$
\left(\Gamma_{a}^{K} \otimes i\right)(\omega)=\omega_{1,3} \omega_{2,3}
$$

and, therefore:

$$
(\Gamma \otimes i)(\Omega)=\Omega_{1,3} \Omega_{2,3}
$$

and, by the same arguments with respect to the second variable, we get:

$$
(i \otimes \Gamma)(\Omega)=\Omega_{1,2} \Omega_{1,3}
$$

4.5. Remark. ([L]) For $\theta$ in $\widehat{K}, t$ in $K$, the function $(\theta, t) \rightarrow\langle\theta, t\rangle$ is an element of $L^{\infty}(\widehat{K}) \otimes L^{\infty}(K)$; via the Fourier-Plancherel isomorphism, we know that $\mathscr{L}(K)$ is isomorphic to $L^{\infty}(\widehat{K})$ (and $\mathscr{L}(\widehat{K})$ to $\left.L^{\infty}(K)\right)$; via this isomorphism, it may be written ([ES2], 4.4.3) as $\int_{K} \lambda_{K}(s)^{*} \otimes d P_{s}$, where $P_{s}$ is a spectral measure on $K$ with values in $\mathscr{L}(\widehat{K})$. Therefore, the function $\omega$ defined in 4.1 , considered, via the Fourier-Plancherel isomorphism, as an element of $\mathscr{L}(\widehat{K}) \otimes \mathscr{L}(\widehat{K})$, may be written as $\int_{K} \lambda_{\widehat{K}}(\pi(s))^{*} \otimes d P_{s}$, and the lifted 2-cocycle $\Omega \in M \otimes M$ is equal to:

$$
\Omega=\int_{K} \alpha\left(\lambda_{\widehat{K}}(\pi(s))\right)^{*} \otimes d\left(\alpha\left(P_{s}\right)\right)
$$

4.6. Definition. ([L]) With the hypothesis of 4.3, we shall say that $\Omega$ is an abelian cocycle of $(M, \Gamma, \kappa)$, constructed with $(K, \pi, \alpha)$.

If $P$ is the support of $\alpha$, then, as, by ([ES2] 1.2.7, 2.2.6 and 4.3.6(iii)), there exists an open subgroup $K^{\prime}$ of $K$ such that $L^{\infty}(K)_{P}$ is isomorphic to $L^{\infty}\left(K^{\prime}\right)$; let $\alpha^{\prime}$ be then the canonical the homomorphism from $L^{\infty}\left(K^{\prime}\right)$ to $M$. As $\hat{K}^{\prime}=\hat{K} / K^{\prime} \perp$, we can then construct a continuous homomorphism $\pi^{\prime}$ from $K^{\prime}$ to $\hat{K}^{\prime}$ such that $\Omega$ is constructed with $\left(K^{\prime}, \pi^{\prime}, \alpha^{\prime}\right)$.

Let us take now $K^{\prime \prime}=K{\overline{\pi^{\prime}\left(K^{\prime}\right)}}^{\perp}$. We have then $\hat{K}^{\prime \prime}=\overline{\pi^{\prime}\left(K^{\prime}\right)}$, and, therefore, the co-involutive Hopf-von Neumann algebra $\left(L^{\infty}\left(K^{\prime \prime}\right), \Gamma_{a}^{K^{\prime \prime}}, \kappa_{a}^{K^{\prime \prime}}\right)$, which is isomorphic to $\left(\mathscr{L}\left(\hat{K}^{\prime \prime}\right), \Gamma_{s}^{\hat{K}^{\prime \prime}}, \kappa_{s}^{\hat{K}^{\prime \prime}}\right)$, is a sub co-involutive Hopf-von Neumann algebra of $\left(\mathscr{L}\left(\hat{K}^{\prime}\right), \Gamma_{s}^{K^{\prime}}, \kappa_{s}^{\hat{K}^{\prime}}\right)$, which is isomorphic to $\left(L^{\infty}\left(K^{\prime}\right), \Gamma_{a}^{K^{\prime}}, \kappa_{a}^{K^{\prime}}\right)$; therefore, if $\Omega$ is an abelian cocycle of $(M, \Gamma, \kappa)$, constructed with $(K, \pi, \alpha)$, we can always suppose that $\alpha$ is injective, and that $\overline{\pi(K)}=\hat{K}$ (or, equivalently, that $\pi$ is injective). 
4.7. Remark. (i) If $\mathbb{W}=(M, \Gamma, \kappa, \tau, \varphi)$ a Woronowicz algebra, with $\varphi$ finite (it comes then, using ([BS], Sect. 4), from a compact quantum group), and if $\Omega$ is an abelian cocycle of $(M, \Gamma, \kappa)$, then $\Omega$ is constructed from $(K, \pi, \alpha)$, with $\alpha$ injective, and $\overline{\pi(K)}=\hat{K}$; then, as $\varphi \circ \alpha$ is a bounded left Haar measure on $K$, we get that $K$ is compact, therefore $\hat{K}=\overline{\pi(K)}=\pi(K)$ also, and so $K$ is finite.

(ii) If $\mathbb{W}=(M, \Gamma, \kappa, \tau, \varphi)$ a Woronowicz algebra, such that the predual $M_{*}$ has a unit $\varepsilon$ (it comes then from a discrete quantum group), and if $\Omega$ is an abelian cocycle of $(M, \Gamma, \kappa)$, then $\Omega$ is constructed from $(K, \pi, \alpha)$, with $\alpha$ injective, and $\overline{\pi(K)}=\hat{K}$; then, as $\varepsilon \circ \alpha$ is a unit of $L^{1}(K)$, we get that $K$ is discrete, therefore $\hat{K}=\overline{\pi(K)}=\pi(K)$ also, and so $K$ is finite.

\section{Abelian Cocycles of Kac Algebras}

5.1. Proposition. Let $(M, \Gamma, \kappa, \varphi)$ be a Kac algebra, $W$ its fundamental unitary, $K$ an abelian locally compact group, $\alpha$ a co-involutive Hopf-von Neumann morphism from $\left(L^{\infty}(K), \Gamma_{a}^{K}, \kappa_{a}^{K}\right)$ to $(M, \Gamma, \kappa)$ such that $\alpha\left(L^{\infty}(K)\right)$ is included in $M^{\varphi}$ (where $\left.M^{\varphi}=\left\{x \in M, \sigma_{t}^{\varphi}(x)=x, \forall t \in \mathbb{R}\right\}\right), \omega$ an involutive cocycle on $K$ as in 4.1 , and $\Omega=(\alpha \otimes \alpha)(\omega)$. Then $\varphi$ is left-invariant with respect to $\Gamma_{\Omega}$, i.e. we have, for all $z$ in $M^{+}$:

$$
(i \otimes \varphi) \Gamma_{\Omega}(z)=\varphi(z) 1 .
$$

Proof. Let $a_{i}, b_{i}$ in $L^{\infty}(K)$ such that $\Sigma_{i}\left(a_{i} \otimes b_{i}\right) \rightarrow_{i} \omega$, and all finite sums of norm less or equal to 1 . Let $x, y$ in $\mathfrak{N}_{\varphi} \cap \mathfrak{N}_{\varphi}^{*}$, and $\xi^{\prime}, \eta^{\prime}$ right bounded vectors with respect to $\varphi$; using 4.3(ii), we get that $\Sigma_{i}\left(\alpha\left(a_{i}\right)^{*} \otimes J \alpha\left(b_{i}\right) J\right)$ is weakly converging to $\tilde{\Omega}$, and, therefore, using that $\alpha\left(L^{\infty}(K)\right)$ lies in $M^{\varphi}$, we get that $W \tilde{\Omega}\left(\Lambda_{\varphi}(x) \otimes \Lambda_{\varphi}(y)\right)$ is the weak limit of

$$
\begin{aligned}
& W \Sigma_{l}\left(\alpha\left(a_{i}\right)^{*} \otimes J \alpha\left(b_{i}\right) J\right)\left(\Lambda_{\varphi}(x) \otimes \Lambda_{\varphi}(y)\right)=W \Sigma_{l}\left(\Lambda_{\varphi}\left(\alpha\left(a_{i}\right)^{*} x\right) \otimes \Lambda_{\varphi}\left(y \alpha\left(b_{i}\right)^{*}\right)\right) \\
& \quad=\Lambda_{\varphi \otimes \varphi}\left(\Gamma(y)(\alpha \otimes \alpha)\left(\Sigma_{i} \Gamma_{a}\left(b_{i}^{*}\right)\left(a_{i}^{*} \otimes 1\right)\right)(x \otimes 1)\right),
\end{aligned}
$$

and therefore we get that $\left(\pi^{\prime}\left(\xi^{\prime}\right) \otimes \pi^{\prime}\left(\eta^{\prime}\right)\right) W \tilde{\Omega}\left(\Lambda_{\varphi}(x) \otimes \Lambda_{\varphi}(y)\right)$ is the weak limit of

$$
\begin{aligned}
& \left(\left(\pi^{\prime}\left(\xi^{\prime}\right) \otimes \pi^{\prime}\left(\eta^{\prime}\right)\right) \Lambda_{\varphi \otimes \varphi}\left(\Gamma(y)(\alpha \otimes \alpha)\left(\Sigma_{i} \Gamma_{a}\left(b_{i}^{*}\right)\left(a_{i}^{*} \otimes 1\right)\right)(x \otimes 1)\right)\right. \\
& \quad=\Gamma(y)(\alpha \otimes \alpha)\left(\Sigma_{l} \Gamma_{a}\left(b_{i}^{*}\right)\left(a_{i}^{*} \otimes 1\right)\right)(x \otimes 1)\left(\xi^{\prime} \otimes \eta^{\prime}\right)
\end{aligned}
$$

and is, using 4.3(i), equal to $\Gamma(y) \Omega^{*}(x \otimes 1)\left(\xi^{\prime} \otimes \eta^{\prime}\right)$. We then deduce that $\Gamma(y) \Omega^{*}(x \otimes 1)$ belongs to $\mathfrak{N}_{\varphi \otimes \varphi}$ and that

$$
\Lambda_{\varphi \otimes \varphi}\left(\Gamma(y) \Omega^{*}(x \otimes 1)\right)=W \tilde{\Omega}\left(\Lambda_{\varphi}(x) \otimes \Lambda_{\varphi}(y)\right)
$$

from which we get

$$
\Lambda_{\varphi \otimes \varphi}\left(\Gamma_{\Omega}(y)(x \otimes 1)\right)=W_{\Omega}\left(\Lambda_{\varphi}(x) \otimes \Lambda_{\varphi}(y)\right)
$$

and, then

$$
\left(\omega_{\Lambda_{\varphi}(x), \Lambda_{\varphi}(x)} \otimes \varphi\right) \Gamma_{\Omega}\left(y^{*} y\right)=(\varphi \otimes \varphi)\left(\left(x^{*} \otimes 1\right) \Gamma_{\Omega}\left(y^{*} y\right)(x \otimes 1)\right)=\varphi\left(x^{*} x\right) \varphi\left(y^{*} y\right) .
$$

As $\Omega$ belongs to $M^{\varphi} \otimes M^{\varphi}$, we have, for all $x$ in $M$, thanks to ([ES2], 2.5.6):

$$
\Gamma_{\Omega}\left(\sigma_{t}^{\varphi}(x)\right)=\Omega \Gamma\left(\sigma_{t}^{\varphi}(x)\right) \Omega^{*}=\Omega\left(i \otimes \sigma_{t}^{\varphi}\right)(\Gamma(x)) \Omega^{*}=\left(i \otimes \sigma_{t}^{\varphi}\right)\left(\Gamma_{\Omega}(x)\right)
$$


and, therefore, the weight $\left(\omega_{\Lambda_{\varphi}(x), \Lambda_{\varphi}(x)} \otimes \varphi\right) \Gamma_{\Omega}$ is invariant by $\sigma_{t}^{\varphi}$, from which, by density, we get, for any $z$ in $M^{+}$:

$$
\left(\omega_{\Lambda_{\varphi}(x), \Lambda_{\varphi}(x)} \otimes \varphi\right) \Gamma_{\Omega}(z)=\varphi\left(x^{*} x\right) \varphi(z)
$$

from which, by density, we deduce easily:

$$
(i \otimes \varphi) \Gamma_{\Omega}(z)=\varphi(z) 1 .
$$

5.2. Theorem. Let $(M, \Gamma, \kappa, \varphi)$ be a Kac algebra, $W$ its fundamental unitary, $K$ an abelian locally compact group, $\alpha$ a co-involutive Hopf-von Neumann morphism from $\left(L^{\infty}(K), \Gamma_{a}^{K}, \kappa_{a}^{K}\right)$ to $(M, \Gamma, \kappa)$ such that $\alpha\left(L^{\infty}(K)\right) \subset M^{\varphi}, \omega$ an involutive cocycle on $K$ as in 4.1 , and $\Omega=(\alpha \otimes \alpha)(\omega)$. Then $\mathbb{K}_{\Omega}=\left(M, \Gamma_{\Omega}, \kappa, \varphi\right)$ is a Kac algebra whose fundamental unitary is $W_{\Omega}$.

Proof. By 5.1, the triple $\left(M, \Gamma_{\Omega}, \varphi\right)$ satisfies the hypothesis of ([ES2], 2.4), $W_{\Omega}$ is its fundamental operator, and, using ([ES2], 2.4.6), we can define, for all $\omega$ in $M_{*}$, $\lambda_{\Omega}(\omega)=(\omega \circ \kappa \otimes i)\left(W_{\Omega}\right)$ such that, for all $x$ in $\mathfrak{N}_{\varphi}$ :

$$
\lambda_{\Omega}(\omega) \Lambda_{\varphi}(x)=\Lambda_{\varphi}\left((\omega \circ \kappa \otimes i) \Gamma_{\Omega}(x)\right)
$$

from which we get, for all $\omega$ in $M_{*}$ :

$$
S_{\varphi} \lambda_{\Omega}(\omega) \subset \lambda_{\Omega}(\bar{\omega}) S_{\varphi} .
$$

As, for all $t$ in $\mathbb{R}, \Delta_{\varphi}^{i t}$ belongs to the center of $\hat{M}$ ([ES2], 4.1 .3 (i)), and $\Omega$ (and $\tilde{\Omega})$ commutes with $1 \otimes \Delta_{\varphi}^{i t}$, we get that $W_{\Omega}$ commutes with $1 \otimes \Delta_{\varphi}^{i t}$, and, therefore, that $\lambda_{\Omega}(\omega)$ commutes with $\Delta_{\varphi}^{i t}$, for all $\omega$ in $M_{*}$ and all $t$ in $\mathbb{R}$, from which we deduce that $J_{\varphi} \lambda_{\Omega}(\omega) J_{\varphi}=\lambda_{\Omega}(\bar{\omega})$.

We then get, using 3.9(ii):

$$
\begin{aligned}
\lambda_{\Omega}(\omega)^{*}= & (\bar{\omega} \circ \kappa \otimes i)\left(W_{\Omega}^{*}\right)=(\bar{\omega} \circ \kappa \otimes i)\left(\left(\hat{J} \otimes J_{\varphi}\right) W_{\Omega}\left(\hat{J} \otimes J_{\varphi}\right)\right) \\
& =J_{\varphi}(\omega \otimes i)\left(W_{\Omega}\right) J_{\varphi}=J_{\varphi} \lambda_{\Omega}(\omega \circ \kappa) J_{\varphi}=\lambda_{\Omega}\left(\omega^{o}\right)
\end{aligned}
$$

and, as $\lambda_{\Omega}$ is involutive, we get the result, by ([ES2], 2.4.6 (iv)).

5.3. Corollary. With the hypothesis of 5.2, let $A$ be the canonical sub- $\mathbb{C}^{*}$-algebra of $M$, which is, with the restrictions of $\Gamma, \kappa, \varphi, a \mathbb{C}^{*}-K a c$ algebra; then, $A$, with the restrictions of $\Gamma_{\Omega}, \kappa, \varphi$, is the $\mathbb{C}^{*}$-Kac algebra canonically associated to $\left(M, \Gamma_{\Omega}, \kappa, \varphi\right)$.

Proof. By 4.4 and ([ES2], 1.5.2, 2.6.5), we get that $\Omega$ is the generator of a nondegenerate representation of the involutive Banach algebra $M_{*}$ in $M$; therefore, by ([EV], 5.1.5), $\Omega$ belongs to the multipliers of $B \otimes A$, where $B$ is the $\mathbb{C}^{*}$-algebra generated by this representation; applying the same argument to $\sigma \Omega^{*} \sigma=\Omega$, we get that this algebra $B$ is a subalgebra of $A$; therefore, $\Omega$ belongs to the multipliers $M(A \otimes A)$ of $A \otimes A$; more precisely, by ([BS], A.3d), we get that $\Omega$ belongs to the subalgebra denoted $M_{L}(A \otimes A)$ in ([EV], 2.1.3). So, for any $x$ on $A$, we get that $\Omega \Gamma(x) \Omega^{*}$ belongs to $M_{L}(A \otimes A)$; so, $A$, with the restrictions of $\Gamma_{\Omega}, \kappa, \varphi$, is a $\mathbb{C}^{*}$ Kac algebra, which is, by $([\mathrm{EV}], 4.3 .4)$, the $\mathbb{C}^{*}$-Kac algebra canonically associated to $\left(M, \Gamma_{\Omega}, \kappa, \varphi\right)$.

5.4. Proposition. With the hypothesis of 5.2, let $\omega_{i}$ be a left approximate unit (if it exists!) of the predual $M_{*}$, which is an algebra whose product is given by:

$$
\omega_{1} * \omega_{2}=\left(\omega_{1} \otimes \omega_{2}\right) \circ \Gamma
$$


then $\omega_{i}$ is still a left approximate unit for the deformed product on $M_{*}$, given by:

$$
\omega_{1} *_{\Omega} \omega_{2}=\left(\omega_{1} \otimes \omega_{2}\right) \circ \Gamma_{\Omega} .
$$

Proof. Thanks to ([ES1], 2.4), we know that there exists $\xi_{i}$ in $H_{\varphi}$ such that $\omega_{i}=\omega_{\xi_{i}}$ and:

$$
\left\|W\left(\xi_{i} \otimes \eta\right)-\xi_{i} \otimes \eta\right\| \rightarrow_{i} 0
$$

for all $\eta$ in $H_{\varphi}$. As, by 4.4, we have $(\Gamma \otimes i)(\Omega)=\Omega_{1,3} \Omega_{2,3}$ which, by ([ES2], 1.5.1), means that $\omega \rightarrow(\omega \otimes i)(\Omega)$ is multiplicative, we deduce that $\left(\omega_{i} \otimes i\right)(\Omega) \rightarrow_{i} 1$, from which we infer that, for all $\eta$ in $H_{\varphi}$, we have:

$$
\left(\Omega\left(\xi_{i} \otimes \eta\right) \mid \xi_{i} \otimes \eta\right) \rightarrow_{i}\|\eta\|^{2}
$$

and

$$
\left\|\Omega\left(\xi_{i} \otimes \eta\right)-\xi_{i} \otimes \eta\right\| \rightarrow_{i} 0 .
$$

By definition of $\tilde{\Omega}$, we get, for all $\omega$ in $M_{*}$ :

$$
(\omega \otimes i)(\tilde{\Omega})=J(\omega \circ \kappa \otimes i)(\Omega) J,
$$

and, therefore, the application $\omega \rightarrow(\omega \otimes i)(\tilde{\Omega})$ is anti-multiplicative, and, with the same arguments, we get that:

$$
\left\|\tilde{\Omega}\left(\xi_{i} \otimes \eta\right)-\xi_{i} \otimes \eta\right\| \rightarrow_{i} 0,
$$

from which we deduce that:

$$
\left\|W_{\Omega}\left(\xi_{i} \otimes \eta\right)-\xi_{i} \otimes \eta\right\| \rightarrow_{i} 0,
$$

and, using ([ES1], 2.4) again, we get the result.

5.5. Corollary. With the hypothesis of 5.2, if the Kac algebra $\widehat{\mathbb{K}}$ is amenable in the sense of ([ES1], 2.5), so is $\widehat{\mathbb{K}_{\Omega}}$.

Proof. Clear, using ([ES1], 2.5) and 5.4.

5.6. Remark. It is possible to prove in the context of Woronowicz algebras a similar result to 5.2, if, in addition, $\alpha\left(L^{\infty}(K)\right) \subset M^{\tau}=\left\{x \in M, \tau_{t}(x)=x, \forall t \in \mathbb{R}\right\}$.

\section{Examples}

6.1. Deformations of a Locally Compact Group Lifted by an Abelian Subgroup ([LR], [L]). Let $G$ be a locally compact group; in [L] are studied the deformations of the coproduct $\Gamma_{s}^{G}$ on $\mathscr{L}(G)$ given by a 2 -cocycle for $\left(\mathscr{L}(G), \Gamma_{s}^{G}\right)$ (in Landstad's terminology, a "dual cocycle on $G$ ") or by a 2-cocycle for $\left(\mathscr{L}(G), \Gamma_{s}^{G}, \kappa_{s}^{G}\right)$ (in Landstad's terminology, a "normalized dual cocycle on $G$ "). The only known method of obtaining these objects is by lifting an ordinary cocycle on $\widehat{H}$, where $H$ is a closed abelian subgroup of $G$, and $\widehat{H}$ its Pontrjagin dual.

In that situation, it is well known that there exists an injective morphism $\alpha$ from the von Neumann algebra $\mathscr{L}(H)$ generated by the left regular representation $\lambda_{H}$ of 
$H$ to the von Neumann algebra $\mathscr{L}(G)$, such that, for every $s$ in $H$, we have

$$
\alpha\left(\lambda_{H}(s)\right)=\lambda_{G}(s)
$$

(see, for example, ([ES2], 5.2.6) and [TT]). It is then clear that $\alpha$ is a co-involutive Hopf-von Neumann algebra morphism from $\left(\mathscr{L}(H), \Gamma_{s}^{H}, \kappa_{s}^{H}\right)$ to $\left(\mathscr{L}(G), \Gamma_{s}^{G}, \kappa_{s}^{G}\right)$; let $\hat{H}$ be the Pontrjagin dual of $H$; then, using the Fourier-Plancherel isomorphism, we know ([ES2], 4.3.7) that the co-involutive Hopf-von Neumann algebras $\left(\mathscr{L}(H), \Gamma_{s}^{H}, \kappa_{s}^{H}\right)$ and $\left(L^{\infty}(\widehat{H}), \Gamma_{a}^{\widehat{H}}, \kappa_{a}^{\widehat{H}}\right)$ are isomorphic; we may then consider that $\alpha$ is a co-involutive Hopf-von Neumann algebra morphism from $\left(L^{\infty}(\widehat{H}), \Gamma_{a}^{\widehat{H}}, \kappa_{a}^{\widehat{H}}\right)$ to $\left(\mathscr{L}(G), \Gamma_{s}^{G}, \kappa_{s}^{G}\right)$.

Let us suppose now that $\Delta_{G}(s)=1$, for all $s$ in $H$, where $\Delta_{G}$ is the modulus of $G$; using 3.2(ii), we get that $\alpha\left(L^{\infty}(\widehat{H})\right)$ is included in $\mathscr{L}(G)^{\varphi_{s}^{G}}$. We are therefore in the conditions of 5.2, and, if $\pi$ is a continuous homomorphism from $\widehat{H}$ to $H$, the function $\omega(s, t)=\langle\pi(s), t\rangle$ is an involutive cocycle on $\widehat{H}, \Omega=(\alpha \otimes \alpha)(\omega)$ is a 2-cocycle for $\left(\mathscr{L}(G), \Gamma_{s}^{G}, \kappa_{s}^{G}\right)$, and $\left(\mathscr{L}(G),\left(\Gamma_{s}^{G}\right)_{\Omega}, \kappa_{s}^{G}, \varphi_{s}^{G}\right)$ is a Kac algebra.

Using 4.5 , we see that $\Omega$ can be written as:

$$
\Omega=\int_{\widehat{H}} \lambda_{G}(\pi(s))^{*} \otimes d\left(\alpha\left(P_{s}\right)\right),
$$

where $P_{s}$ is the spectral measure on $\widehat{H}$, with values in $\mathscr{L}(H)$ such that the operator $\int_{\widehat{H}} \lambda_{\widehat{H}}(s)^{*} \otimes d P_{s}$ is the function $(t, s) \rightarrow\langle t, s\rangle$ on $H \times \widehat{H}$, viewed, via the FourierPlancherel isomorphism, as an element of $\mathscr{L}(\widehat{H}) \otimes \mathscr{L}(H)$.

So, using Landstad's terminology and notations, when the normalized dual cocycle $\omega$ on $G$ is lifted from an abelian subgroup $H$ on which the modulus function of $G$ is equal to 1 , the algebra $A\left(G, \delta_{\omega}\right)$ ) is the predual of a Kac algebra; in that situation, we can answer positively to one open problem of [L]; more precisely, by ([ES2], 3.1.4), every representation of this algebra $A\left(G, \delta_{\omega}\right)$ has a generator (in Landstad's terminology, is given by slicing a $\delta_{\omega}$-corepresentation).

\subsection{Deformations of a Semi-Direct Product of Abelian Groups. As a particular} case of the preceding example, we consider $A$ and $H$ locally compact groups and $\alpha$ a continuous action of $H$ on $A$, i.e. a homomorphism $H \rightarrow$ Aut $A$, such that the mapping $(a, s) \rightarrow \alpha_{s}(a)$ is continuous from $A \times H$ to $A$; the semi-direct product $G=A \triangleleft_{\alpha} H$ is the set $A \times H$, equipped with the product

$$
(a, s)(b, t)=\left(a \alpha_{s}(b), s t\right) .
$$

It is a locally compact group, whose right Haar measure is the product of right Haar measures on $A$ and $H$; the modulus function $\Delta_{A>\triangleleft_{\alpha} H}$ is given by:

$$
\Delta_{A>\triangleleft_{\alpha} H}(a, s)=\delta(s) \Delta_{A}(a) \Delta_{H}(s),
$$

where $\delta(s)$ is the modulus of the automorphism $\alpha_{s}$.

We can consider $A$ as a closed subgroup of $G$, and, if $A$ is abelian, the pair $(G, A)$ satisfies then the conditions of 6.1. If, moreover, $H$ is amenable (for instance if $H$ is abelian), then it is known that the semi-direct product is amenable.

We can also consider $H$ as a closed subgroup of $G$. If $H$ is abelian, and if the modulus $\delta$ is equal to 1 , i.e. if the action of $H$ leaves invariant the Haar measure on 
$A$, we are again in the conditions of 6.1. If, moreover, $A$ is amenable (for instance if $A$ is abelian), the semi-direct product is again amenable.

6.3. Quantizations of the Heisenberg Group. As a specific example, we can take the usual Heisenberg group $H_{n}(\mathbb{R})$, which can be considered as the semi-direct product $\mathbb{R}^{n+1}>\triangleleft_{\alpha} \mathbb{R}^{n}$, where the action $\alpha$ of $\mathbb{R}^{n}$ on $\mathbb{R}^{n+1}$ is given, for $a, b$ in $\mathbb{R}^{n}$, $t$ in $\mathbb{R}$, by:

$$
\alpha_{a}(b, t)=(b, t+(a \mid b)) .
$$

Therefore, the product rule in $H_{n}(\mathbb{R})$ is given by $\left(a, a^{\prime}, b, b^{\prime}\right.$ in $\mathbb{R}^{n}, t, t^{\prime}$ in $\left.\mathbb{R}\right)$ :

$$
(b, t, a)\left(b^{\prime}, t^{\prime}, a^{\prime}\right)=\left(b+b^{\prime}, t+t^{\prime}+\left(a \mid b^{\prime}\right), a+a^{\prime}\right),
$$

and, as the action $\alpha$ leaves the Lebesgue measure of $\mathbb{R}^{n}$ invariant, we are in both situations described in 6.2. The group $H_{n}(\mathbb{R})$ is unimodular, and the Hilbert space $L^{2}\left(H_{n}(\mathbb{R})\right)$ can be identified with $L^{2}\left(\mathbb{R}^{n}\right) \otimes L^{2}(\mathbb{R}) \otimes L^{2}\left(\mathbb{R}^{n}\right)$. So, we get that the left regular representation $\lambda(b, t, a)$ of $H_{n}(\mathbb{R})$ is defined by

$$
\lambda(b, t, a) f(v, u, w)=f(v-b, u-t-(a \mid v-b), w-a),
$$

where $u$ belongs to $\mathbb{R}, v, w$ to $\mathbb{R}^{n}, f$ to $L^{2}\left(\mathbb{R}^{n}\right) \otimes L^{2}(\mathbb{R}) \otimes L^{2}\left(\mathbb{R}^{n}\right)$. Let us define a unitary $U$ on that Hilbert space by

$$
U f(\hat{v}, \hat{u}, w)=(|\hat{u}| / 2)^{1 / 2} \int_{\mathbb{R}^{n} \times \mathbb{R}} f(v, u, \hat{v}-w) e^{l(v \mid \hat{v}) \hat{u}} e^{\imath u \hat{u}} d u d v
$$

where $u, \hat{u}$ belong to $\mathbb{R}, v, w, \hat{v}$ to $\mathbb{R}^{n}, f$ to $L^{2}\left(\mathbb{R}^{n}\right) \otimes L^{2}(\mathbb{R}) \otimes L^{2}\left(\mathbb{R}^{n}\right)$ and we get that the left regular representation $\lambda(b, t, a)$ of $H_{n}(\mathbb{R})$ verifies:

$$
U \lambda(b, t, a) U^{*} f(v, u, w)=e^{i(b \mid v) u} e^{i t u} f(v+a, u, w) .
$$

Therefore, this representation is equivalent to the representation $\pi$ on $L^{2}\left(\mathbb{R}^{n+1}\right)$ defined, for any $\phi$ in $L^{2}\left(\mathbb{R}^{n+1}\right)$, by:

$$
\pi(b, t, a) \phi(u, v)=e^{i(b \mid v) u} e^{i t u} \phi(u, v+a) .
$$

This representation generates the von Neumann algebra $L^{\infty}(\mathbb{R}) \otimes \mathscr{L}\left(L^{2}\left(\mathbb{R}^{n}\right)\right)$, which is therefore isomorphic to $\mathscr{L}\left(H_{n}(\mathbb{R})\right)$; by this isomorphism, there exists a symmetric Kac algebra $\left(\epsilon^{\infty}(\mathbb{R}) \otimes \mathscr{L}\left(L^{2}\left(\mathbb{R}^{n}\right)\right), \Gamma_{s}, \kappa_{s}, \varphi_{s}\right)$, such that:

$$
\begin{aligned}
& \Gamma_{s}(\pi(b, t, a))=\pi(b, t, a) \otimes \pi(b, t, a), \\
& \kappa_{s}(\pi(b, t, a))=\pi(-b,-t+(a \mid b),-a) .
\end{aligned}
$$

As we have

$$
\pi(b, t, 0)=\left((u, v) \rightarrow e^{i(b \mid v) u} e^{i t u}\right),
$$

the morphism $\beta_{1}$ from $L^{\infty}\left(\widehat{\mathbb{R}^{n+1}}\right)$ into $L^{\infty}(\mathbb{R}) \otimes \mathscr{L}\left(L^{2}\left(\mathbb{R}^{n}\right)\right)$ we get from the inclusion $\mathbb{R}^{n+1} \subset H_{n}(\mathbb{R})$, sends the function $(u, v) \rightarrow e^{i t u} e^{i(b \mid v)}$ on $\pi(b, t, 0)$, and, therefore, for any $f$ in $L^{\infty}\left(\widehat{\mathbb{R}^{n+1}}\right), \beta_{1}(f)$ is the function $(u, v) \rightarrow f(u, u v)$.

As $\pi(0,0, a)=1 \otimes \rho(a)$, where $\rho$ is the right regular representation of $\mathbb{R}^{n}$, the morphism $\beta_{2}$ from $L^{\infty}\left(\widehat{\mathbb{R}^{n}}\right)$ into $E^{\infty}(\mathbb{R}) \otimes \mathscr{L}\left(L^{2}\left(\mathbb{R}^{n}\right)\right)$ we get from the inclusion $\mathbb{R}^{n} \subset H_{n}(\mathbb{R})$, is given by:

$$
\beta_{2}(f)=1 \otimes F f F^{*},
$$

where $F$ is the Fourier-Plancherel unitary from $L^{2}\left(\widehat{\mathbb{R}^{n}}\right)$ to $L^{2}\left(\mathbb{R}^{n}\right)$. 
Using 4.2, we can construct, for all $j, k \in\{1, \ldots, n\}, j \neq k$, and $q_{j k}$ in $\mathbb{R}$, a 2-cocycle $\omega_{j k}$ on $\widehat{\mathbb{R}^{n+1}}$ by the formula $\omega_{j k}\left(\hat{u}, \hat{v}, \hat{u^{\prime}}, \hat{v^{\prime}}\right)=e^{i q_{j k}\left(\hat{v_{j}} v_{k}^{\prime}-v_{k} \hat{v}_{j}^{\prime}\right)}$, where $\hat{u}, \hat{u^{\prime}}$ belong to $\widehat{\mathbb{R}}, \hat{v}, \hat{v^{\prime}}$ belong to $\widehat{\mathbb{R}^{n}}$, and $\hat{v_{i}}$ (resp. $\hat{v_{i}^{\prime}}$ ) is the $i^{\text {th }}$ component of $\hat{v}$ (resp. $\left.\hat{v}^{\prime}\right)$. So, $\Omega_{j k}=\left(\beta_{1} \otimes \beta_{1}\right)\left(\omega_{j k}\right)$ is a 2 -cocycle for $\left(E^{\infty}(\mathbb{R}) \otimes \mathscr{L}\left(L^{2}\left(\mathbb{R}^{n}\right)\right), \Gamma_{s}, \kappa_{s}\right)$, and $\Omega_{j k}$ is the function on $\mathbb{R}^{n+1} \times \mathbb{R}^{n+1}$ given by:

$$
\Omega_{j k}\left(u, v, u^{\prime}, v^{\prime}\right)=e^{i q_{j k} u u^{\prime}\left(v_{j} v_{k}^{\prime}-v_{k} v_{j}^{\prime}\right)},
$$

and we get a deformed coproduct $\Gamma_{j k}$ defined by:

$$
\Gamma_{j k}(\pi(b, t, a))=\Omega_{j k}(\pi(b, t, a) \otimes \pi(b, t, a)) \Omega_{j k}^{*},
$$

and we obtain:

$$
\begin{aligned}
\Gamma_{j k}(\pi(b, t, a)) f\left(u, v, u^{\prime}, v^{\prime}\right)= & e^{-i q_{j k} u u^{\prime} a_{k}\left(v_{J}-v_{j}^{\prime}\right)} e^{i q_{j k} u u^{\prime} a_{J}\left(v_{k}-v_{k}^{\prime}\right)} \\
& e^{i(v \mid b) u} e^{i\left(v^{\prime} \mid b\right) u^{\prime}} e^{i t u} e^{i t u^{\prime}} f\left(u, v+a, u^{\prime}, v^{\prime}+a\right),
\end{aligned}
$$

where $u, u^{\prime}$ belong to $\mathbb{R}, v, v^{\prime}$ to $\mathbb{R}^{n}, a_{l}$ is the $\mathrm{i}^{\text {th }}$ component of $a$, and $f$ belongs to $L^{2}\left(\mathbb{R}^{n+1} \times \mathbb{R}^{n+1}\right)$. These coproducts are clearly non symmetric. This construction gives new Kac algebras $\left(t^{\infty}(\mathbb{R}) \otimes \mathscr{L}\left(L^{2}\left(\mathbb{R}^{n}\right)\right), \Gamma_{j k}, \kappa_{s}, \varphi_{s}\right)$, whose duals are amenable. In fact, taking into account the parameter $q_{i j}$ we obtain a one-parameter quantization of the Heisenberg group (if $n \geqq 2$; for $n=1$, the cocycle is trivial, and there is no deformation). Of course, for $n \geqq 3$, it is possible to deal with a cocycle which is the product of such cocycles, in order to get multiparameter quantizations of the Heisenberg group. For instance, if $n=3$, we can construct a 3-parameter (with parameters $q_{12}, q_{23}, q_{31}$ ) quantization, and, for any $n$, we get a quantization parametrized by the vector space of $n$ by $n$ antisymmetric matrices over $\mathbb{R}$, i.e. by $\mathbb{R}^{n(n-1) / 2}$.

Using 4.2 again], we can construct, for all $j, k \in\{1, \ldots, n\}, j \neq k$, and $q_{j k}$ in $\mathbb{R}$, a 2-cocycle $\omega^{j k}$ on $\widehat{\mathbb{R}^{n}}$ by the formula $\omega^{j k}\left(\hat{v}, \hat{v^{\prime}}\right)=e^{i q_{j k}\left(\hat{v}_{j} v_{k}^{\hat{j}}-v_{k} \hat{v}_{J}^{\prime}\right)}$, where $\hat{v}, \hat{v^{\prime}}$ belong to $\widehat{\mathbb{R}^{n}}$, and $\hat{v_{i}}$ (resp. $\hat{v_{i}^{\prime}}$ ) is the $i^{\text {th }}$ component of $\hat{v}$ (resp. $\hat{v^{\prime}}$ ); let us put $\Omega^{j k}=\left(\beta_{2} \otimes \beta_{2}\right)\left(\omega^{j k}\right)$; so $\Omega^{j k}$ is a 2-cocycle for $\left(E^{\infty}(\mathbb{R}) \otimes \mathscr{L}\left(L^{2}\left(\mathbb{R}^{n}\right)\right), \Gamma_{s}, \kappa_{s}\right)$, and this leads to a deformed coproduct $\Gamma^{j k}$ such as:

$$
\Gamma^{j k}(\pi(b, t, a))=\Omega^{j k}(\pi(b, t, a) \otimes \pi(b, t, a)) \Omega^{j k *} .
$$

We get that:

$$
\left(1 \otimes F^{*}\right) \pi(b, t, a)(1 \otimes F) \phi(u, \hat{v})=e^{i t u} e^{i(a \mid \hat{v})} \phi(u, \hat{v}-b u)
$$

for any $\phi$ in $L^{2}\left(\mathbb{R} \times \mathbb{R}^{n}\right)$, from which we get that:

$$
\begin{gathered}
(1 \otimes F \otimes 1 \otimes F) \Gamma^{j k}(\pi(b, t, a)) \phi\left(u, \hat{v}, u^{\prime}, \hat{v^{\prime}}\right)=e^{i t u} e^{i t u^{\prime}} e^{i(a \mid \hat{v})} e^{i\left(a \mid v^{\prime}\right)} \\
e^{\imath q_{j k} u^{\prime}\left(b_{k} \hat{v_{j}}-b_{j} \hat{v}_{k}\right)} e^{\imath q_{j k} u\left(b_{j} v_{k}^{\hat{v}}-b_{k} v_{j}^{\prime}\right)} \phi\left(u, \hat{v}-b u, u^{\prime}, \hat{v^{\prime}}-b u\right)
\end{gathered}
$$

for any $\phi$ in $L^{2}\left(\mathbb{R} \times \widehat{\mathbb{R}^{n}} \times \mathbb{R} \times \widehat{\mathbb{R}^{n}}\right)$, and, using Fourier transform again, we get:

$$
\begin{aligned}
& \Gamma^{j k}(\pi(b, t, a)) f\left(u, v, u^{\prime}, v^{\prime}\right)=e^{i(v \mid b) u} e^{i\left(v^{\prime} \mid b\right) u^{\prime}} e^{i t u} e^{i t u^{\prime}} \\
& \quad f\left(u, v+u^{\prime} q_{j k}\left(b_{k} \varepsilon_{j}-b_{j} \varepsilon_{k}\right)+a, u^{\prime}, v^{\prime}-u q_{j k}\left(b_{k} \varepsilon_{j}-b_{j} \varepsilon_{k}\right)+a\right),
\end{aligned}
$$


where $u, u^{\prime}$ belong to $\mathbb{R}, v, v^{\prime}$ to $\mathbb{R}^{n}, b_{i}$ is the $\mathrm{i}^{\text {th }}$ component of $b,\left(\varepsilon_{i}\right)_{i}$ the canonical basis of $\mathbb{R}^{n}$, and $f$ belongs to $L^{2}\left(\mathbb{R}^{n+1} \times \mathbb{R}^{n+1}\right)$. This coproduct is clearly non-symmetric, and this construction gives again Kac algebras $\left(L^{\infty}(\mathbb{R}) \otimes \mathscr{L}\left(L^{2}\left(\mathbb{R}^{n}\right)\right), \Gamma^{j k}, \kappa_{s}, \varphi_{s}\right)$.

In fact, we shall see that these Kac algebras are isomorphic to the previous ones: let us consider now the involutive automorphism $\gamma$ of $H_{n}(\mathbb{R})$ given by:

$$
\gamma(b, t, a)=(a,-t+(a \mid b), b) .
$$

Using ([ES2], 5.1.4 and 5.3.3), we can construct an involutive automorphism $K_{s}(\gamma)$ of $\mathcal{L}^{\infty}(\mathbb{R}) \otimes \mathscr{L}\left(L^{2}\left(\mathbb{R}^{n}\right)\right)$ such that:

$$
\begin{gathered}
K_{s}(\gamma)(\pi(b, t, a))=\pi(\gamma(b, t, a)), \\
\Gamma_{s} \circ K_{s}(\gamma)=\left(K_{s}(\gamma) \otimes K_{s}(\gamma)\right) \circ \Gamma_{s}, \\
\kappa_{s} \circ K_{s}(\gamma)=K_{s}(\gamma) \circ \kappa_{s}, \\
\varphi_{s} \circ K_{s}(\gamma)=\varphi_{s} .
\end{gathered}
$$

Moreover, as $\gamma(0,0, a)=(a, 0,0)$, we get that, for any $f$ in $L^{\infty}\left(\widehat{\mathbb{R}^{n}}\right)$, we have

$$
K_{s}(\gamma) \circ \beta_{2}(f)=\beta_{1}(\tilde{f}),
$$

where $\tilde{f}$ is the element of $L^{\infty}\left(\widehat{\mathbb{R}^{n+1}}\right)$ such that $\tilde{f}(\hat{u}, \hat{v})=f(\hat{v})$, for any $\hat{u}$ in $\hat{\mathbb{R}}, \hat{v}$ in $\widehat{\mathbb{R}^{n}}$. From which we infer that, for all $j, k, \Omega_{j k}=\left(K_{s}(\gamma) \otimes K_{s}(\gamma)\right)\left(\Omega^{j k}\right)$, and

$$
\Gamma_{j k} \circ K_{s}(\gamma)=\left(K_{s}(\gamma) \otimes K_{s}(\gamma)\right) \circ \Gamma^{j k},
$$

and, therefore, we get that $K_{s}(\gamma)$ is an isomorphism between the Kac algebras $\left(L^{\infty}(\mathbb{R}) \otimes \mathscr{L}\left(L^{2}\left(\mathbb{R}^{n}\right)\right), \Gamma^{j k}, \kappa_{s}, \varphi_{s}\right)$ and $\left(L^{\infty}(\mathbb{R}) \otimes \mathscr{L}\left(L^{2}\left(\mathbb{R}^{n}\right)\right), \Gamma_{j k}, \kappa_{s}, \varphi_{s}\right)$.

We can consider the Lie algebra $L$ of the Heisenberg group $H_{n}(\mathbb{R})$, and define, where $u$ belongs to $\mathbb{R}, v$ to $\mathbb{R}^{n}, v_{k}$ is the $\mathrm{k}^{\text {th }}$ component of $v$, and $\phi$ is in the Schwartz algebra $\mathscr{S}\left(\mathbb{R}^{n+1}\right)$, its generators $P_{k}, R, Q_{k}(k \in\{1, \ldots, n\})$ by

$$
\begin{aligned}
P_{k} \phi(u, v) & =\left.\frac{\partial}{\partial a_{k}}[\pi(b, t, a) \phi(u, v)]\right|_{a=0, b=0, t=0}=\frac{\partial \phi}{\partial v_{k}}(u, v), \\
R \phi(u, v) & =\left.\frac{\partial}{\partial t}[\pi(b, t, a) \phi(u, v)]\right|_{a=0, b=0, t=0}=i u \phi(u, v) \\
Q_{k} \phi(u, v) & =\left.\frac{\partial}{\partial b_{k}}[\pi(b, t, a) \phi(u, v)]\right|_{a=0, b=0, t=0}=i u v_{k} \phi(u, v),
\end{aligned}
$$

which are the infinitesimal operators of the representation $\pi$. These operators are linked by the commutations relations $\left[P_{k}, R\right]=\left[Q_{k}, R\right]=0$ and $\left[P_{k}, Q_{j}\right]=\delta_{j k} R$.

The symmetric coproduct $\Gamma_{S}$ of the envelopping algebra $U(L)$ satisfies

$$
\begin{gathered}
\Gamma_{s}\left(P_{k}\right)=P_{k} \otimes 1+1 \otimes P_{k}, \\
\Gamma_{s}(R)=R \otimes 1+1 \otimes R, \\
\Gamma_{s}\left(Q_{k}\right)=Q_{k} \otimes 1+1 \otimes Q_{k},
\end{gathered}
$$

the antipode $\kappa_{s}$ and the involution $*$ verify

$$
\kappa_{s}\left(P_{k}\right)=P_{k}^{*}=-P_{k}, \kappa_{s}(R)=R^{*}=-R, \kappa_{s}\left(Q_{k}\right)=Q_{k}^{*}=-Q_{k} .
$$


The first quantization leads, for $l \neq k$ and $l \neq j$, and $m \in\{1, . ., n\}$, to:

$$
\begin{gathered}
\Gamma_{j k}\left(P_{l}\right)=P_{l} \otimes 1+1 \otimes P_{l}, \\
\Gamma_{j k}\left(P_{j}\right)=P_{j} \otimes 1+1 \otimes P_{j}-q_{j k}\left(Q_{k} \otimes i R-i R \otimes Q_{k}\right), \\
\Gamma_{j k}\left(P_{k}\right)=P_{k} \otimes 1+1 \otimes P_{k}+q_{j k}\left(Q_{j} \otimes i R-i R \otimes Q_{j}\right), \\
\Gamma_{j k}(R)=R \otimes 1+1 \otimes R, \\
\Gamma_{j k}\left(Q_{m}\right)=Q_{m} \otimes 1+1 \otimes Q_{m} .
\end{gathered}
$$

The second quantization leads, for $l \neq k$ and $l \neq j$, and $m \in\{1, \ldots, n\}$, to:

$$
\begin{gathered}
\Gamma^{j k}\left(P_{m}\right)=P_{m} \otimes 1+1 \otimes P_{m}, \\
\Gamma^{j k}(R)=R \otimes 1+1 \otimes R, \\
\Gamma^{j k}\left(Q_{l}\right)=Q_{l} \otimes 1+1 \otimes Q_{l}, \\
\Gamma^{j k}\left(Q_{k}\right)=Q_{k} \otimes 1+1 \otimes Q_{k}-q_{j k}\left(P_{j} \otimes i R-i R \otimes P_{j}\right), \\
\Gamma^{j k}\left(Q_{j}\right)=Q_{j} \otimes 1+1 \otimes Q_{j}+q_{j k}\left(P_{k} \otimes i R-i R \otimes P_{k}\right) .
\end{gathered}
$$

We obtain here two "quantum groups" in the sense of [D], which are clearly isomorphic.

6.4. Kac-Paljutkin's Dimension 8 example [KP2]. This "historic" example of nontrivial (i.e. non commutative and non-symmetric) finite dimensional Kac algebra (i.e. finite dimensional semi-simple $*$-Hopf algebra) is due to Kac and Paljutkin, published in Russian in 1965. A description of this example is done in ([BS], 8.26.1); another description, using our constructions, is the following: let us start with the group $G$ equal to the semi-direct product $\left(\mathbb{Z}_{2}\right)^{2} \triangleleft_{\alpha} \mathbb{Z}_{2}$, where the action $\alpha$ of $\mathbb{Z}_{2}$ is just the permutation of the generators $a$ and $b$ of $\left(\mathbb{Z}_{2}\right)^{2}$. So, the group $G$ has eight elements

$$
G=\{1, a, b, a b=b a, s, a s=s b, b s=s a, a b s=s a b\}
$$

and the subgroup $\{1, a, b, a b\}$ is isomorphic to $\left(\mathbb{Z}_{2}\right)^{2}$. On the group algebra $\mathscr{L}(G)$ (3.2(ii)) a structure of Kac algebra can be put; moreover, as it is dimension 8, and as there is a projection of dimension 1 in the center of $\mathscr{L}(G)$ ([ES2], 6.3.5), and as it is not abelian (because $G$ is not abelian) we get that $\mathscr{L}(G)$ is isomorphic to $\mathbb{C} \oplus \mathbb{C} \oplus \mathbb{C} \oplus \mathbb{C} \oplus M_{2}(\mathbb{C})$; let $e_{1}, e_{2}, e_{3}, e_{4}, e_{11}, e_{12}, e_{21}, e_{22}$ be the matrix units of this algebra; therefore, up to an isomorphism, we can write the left regular representation $\lambda$ of $G$ as the following:

$$
\begin{gathered}
\lambda(1)=e_{1}+e_{2}+e_{3}+e_{4}+e_{11}+e_{22}, \\
\lambda(a)=e_{1}-e_{2}+e_{3}-e_{4}-e_{11}+e_{22}, \\
\lambda(b)=e_{1}-e_{2}+e_{3}-e_{4}+e_{11}-e_{22}, \\
\lambda(a b)=e_{1}+e_{2}+e_{3}+e_{4}-e_{11}-e_{22}, \\
\lambda(s)=e_{1}+e_{2}-e_{3}-e_{4}+e_{12}+e_{21}, \\
\lambda(a s)=e_{1}-e_{2}-e_{3}+e_{4}-e_{12}+e_{21}, \\
\lambda(b s)=e_{1}-e_{2}-e_{3}+e_{4}+e_{12}-e_{21}, \\
\lambda(a b s)=e_{1}+e_{2}-e_{3}-e_{4}-e_{12}-e_{21},
\end{gathered}
$$


from which we can get formulas for the symmetric coproduct $\Gamma_{s}$ on $\mathscr{L}(G)$ :

$$
\begin{aligned}
\Gamma_{s}\left(e_{1}\right)= & e_{1} \otimes e_{1}+e_{2} \otimes e_{2}+e_{3} \otimes e_{3}+e_{4} \otimes e_{4} \\
& +1 / 2\left(e_{11} \otimes e_{11}+e_{22} \otimes e_{22}+e_{12} \otimes e_{12}+e_{21} \otimes e_{21}\right), \\
\Gamma_{s}\left(e_{2}\right)= & e_{1} \otimes e_{2}+e_{2} \otimes e_{1}+e_{3} \otimes e_{4}+e_{4} \otimes e_{3} \\
& +1 / 2\left(e_{11} \otimes e_{22}+e_{22} \otimes e_{11}+e_{12} \otimes e_{21}+e_{21} \otimes e_{12}\right), \\
\Gamma_{s}\left(e_{3}\right)= & e_{1} \otimes e_{3}+e_{2} \otimes e_{4}+e_{3} \otimes e_{1}+e_{4} \otimes e_{2} \\
& +1 / 2\left(e_{11} \otimes e_{11}+e_{22} \otimes e_{22}-e_{12} \otimes e_{12}-e_{21} \otimes e_{21}\right), \\
\Gamma_{s}\left(e_{4}\right)= & e_{1} \otimes e_{4}+e_{2} \otimes e_{3}+e_{3} \otimes e_{2}+e_{4} \otimes e_{1} \\
& +1 / 2\left(e_{11} \otimes e_{22}+e_{22} \otimes e_{11}-e_{12} \otimes e_{21}-e_{21} \otimes e_{12}\right), \\
\Gamma_{s}\left(e_{11}\right)= & e_{1} \otimes e_{11}+e_{2} \otimes e_{22}+e_{3} \otimes e_{11}+e_{4} \otimes e_{22}+e_{11} \otimes e_{1}+e_{22} \otimes e_{2} \\
& +e_{11} \otimes e_{3}+e_{22} \otimes e_{4}, \\
\Gamma_{s}\left(e_{12}\right)= & e_{1} \otimes e_{12}+e_{2} \otimes e_{21}-e_{3} \otimes e_{12}-e_{4} \otimes e_{21}+e_{12} \otimes e_{1}+e_{21} \otimes e_{2} \\
& -e_{12} \otimes e_{3}-e_{21} \otimes e_{4}, \\
\Gamma_{s}\left(e_{21}\right)= & e_{1} \otimes e_{21}+e_{2} \otimes e_{12}-e_{3} \otimes e_{21}-e_{4} \otimes e_{12}+e_{21} \otimes e_{1}+e_{12} \otimes e_{2} \\
& -e_{21} \otimes e_{3}-e_{12} \otimes e_{4}, \\
& +e_{22} \otimes e_{3}+e_{11} \otimes e_{4}, \\
\Gamma_{s}\left(e_{22}\right)= & e_{1} \otimes e_{22}+e_{2} \otimes e_{11}+e_{3} \otimes e_{22}+e_{4} \otimes e_{11}+e_{22} \otimes e_{1}+e_{11} \otimes e_{2} \\
&
\end{aligned}
$$

and for the involution $*$ and the co-involution $\kappa_{s}$ :

$$
\begin{gathered}
\kappa_{s}\left(e_{i}\right)=e_{i}=e_{i}^{*}(i=1, \ldots, 4), \\
\kappa_{s}\left(e_{11}\right)=e_{11}=e_{11}^{*}, \quad \kappa_{s}\left(e_{22}\right)=e_{22}=e_{22}^{*}, \\
\kappa_{s}\left(e_{12}\right)=e_{21}=e_{12}^{*}, \quad \kappa_{s}\left(e_{21}\right)=e_{12}=e_{21}^{*},
\end{gathered}
$$

and we see, therefore, that $e_{1}$ is the projection given by the co-unit of $\mathscr{L}(G)$ ([ES2], 6.3.5).

The subalgebra $\mathscr{L}\left(\left(\mathbb{Z}_{2}\right)^{2}\right)$ generated by $(\lambda(1), \lambda(a), \lambda(b), \lambda(a b))$, is generated also by $\left(e_{1}+e_{3}, e_{2}+e_{4}, e_{11}, e_{22}\right)$; by the Fourier transform, it is isomorphic to the algebra of functions on $\left(\widehat{\left.\mathbb{Z}_{2}\right)^{2}}\right.$, and this isomorphism sends $e_{1}+e_{3}$ on $\delta_{1}, e_{2}+e_{4}$ on $\delta_{a b}, e_{11}$ on $\delta_{a}$ and $e_{22}$ on $\delta_{b}$ (where $\delta_{x}$ means the Dirac function on $x$, for any $x$ in $\left(\widehat{\left.\mathbb{Z}_{2}\right)^{2}}\right)$.

Let $\omega$ be the function on $\left(\widehat{\left.\mathbb{Z}_{2}\right)^{2}} \times\left(\widehat{\left.\mathbb{Z}_{2}\right)^{2}}\right.\right.$ defined by $\omega(a, b)=\omega(b, a b)=\omega(a b, a)$ $=e^{i \pi / 4}, \omega(1, u)=\omega(u, u)=1, \omega(u, t)=\overline{\omega(t, u)}$ for all $u, t$ in $\widehat{\left(\mathbb{Z}_{2}\right)^{2}}$, and let $\Omega$ be the unitary element of $\mathscr{L}\left(\left(\mathbb{Z}_{2}\right)^{2}\right) \otimes \mathscr{L}\left(\left(\mathbb{Z}_{2}\right)^{2}\right)$ obtained by Fourier tranform. We get

$$
\begin{aligned}
\Omega= & \left(e_{1}+e_{3}\right) \otimes I+e_{11} \otimes\left(e_{1}+e_{3}+e_{11}+e^{i \pi / 4} e_{22}+e^{-i \pi / 4}\left(e_{2}+e_{4}\right)\right) \\
& +e_{22} \otimes\left(e_{1}+e_{3}+e^{-i \pi / 4} e_{11}+e_{22}+e^{i \pi / 4}\left(e_{2}+e_{4}\right)\right) \\
& +\left(e_{2}+e_{4}\right) \otimes\left(e_{1}+e_{3}+e_{2}+e_{4}+e^{\imath \pi / 4} e_{11}+e^{-i \pi / 4} e_{22}\right) .
\end{aligned}
$$


If we consider the function $\left(s_{1}, s_{2}, s_{3}\right) \rightarrow \overline{\omega\left(s_{2}, s_{3}\right) \omega\left(s_{1}, s_{2} s_{3}\right)} \omega\left(s_{1}, s_{2}\right) \omega\left(s_{1} s_{2}, s_{3}\right)$, it takes value 1 except if $s_{1}=s_{3}=a$ (resp. $b, a b$ ), and $s_{2} \neq s_{1}, s_{2} \neq 1$, for which it takes value -1 ; therefore we get

$$
\begin{aligned}
\partial_{2} \Omega= & I \otimes I \otimes I-2 e_{11} \otimes e_{22} \otimes e_{11}-2 e_{22} \otimes e_{11} \otimes e_{22}-2 e_{11} \otimes\left(e_{2}+e_{4}\right) \otimes e_{11} \\
& -2 e_{22} \otimes\left(e_{2}+e_{4}\right) \otimes e_{22}-2\left(e_{2}+e_{4}\right) \otimes e_{11} \otimes\left(e_{2}+e_{4}\right) \\
& -2\left(e_{2}+e_{4}\right) \otimes e_{22} \otimes\left(e_{2}+e_{4}\right),
\end{aligned}
$$

from which we can easily verify that $\partial_{2} \Omega$ commutes with $\lambda(s) \otimes \lambda(s) \otimes \lambda(s)$, and is, therefore, a pseudo- 2 cocycle for $\left(\mathscr{L}(G), \Gamma_{s}\right)$. So, $\left(\mathscr{L}(G),\left(\Gamma_{s}\right)_{\Omega}\right)$ is a Hopf-von Neumann algebra; as the function $\omega$ verifies $\omega(u, t)=\omega\left(u^{-1}, t^{-1}\right)=\overline{\omega(t, u)}$ for all $u, t$ in $\left(\widehat{\left.\mathbb{Z}_{2}\right)^{2}}\right.$, we get that $\left(\mathscr{L}(G),\left(\Gamma_{s}\right)_{\Omega}, \kappa_{s}\right)$ is a co-involutive Hopf-von Neumann algebra. Moreover, we easily get that $\Omega\left(e_{1} \otimes x\right)=\left(e_{1} \otimes x\right) \Omega=e_{1} \otimes x$, for all $x$ in $\mathscr{L}(G)$; from which we infer that $e_{1}$ still gives a co-unit for the new structure, and, as we have $\left(\Gamma_{s}\right)_{\Omega}\left(e_{1}\right)=\Gamma_{s}\left(e_{1}\right)$, we get, by ([ES2], 6.3.5), that it is a Kac algebra.

We obtain then also $\left(\Gamma_{s}\right)_{\Omega}\left(e_{3}\right)=\Gamma_{s}\left(e_{3}\right), \quad\left(\Gamma_{s}\right)_{\Omega}\left(e_{11}\right)=\Gamma_{s}\left(e_{11}\right), \quad\left(\Gamma_{s}\right)_{\Omega}\left(e_{22}\right)=$ $\Gamma_{s}\left(e_{22}\right)$, and, for the other generators:

$$
\begin{aligned}
& \left(\Gamma_{s}\right)_{\Omega}\left(e_{2}\right)=e_{1} \otimes e_{2}+e_{2} \otimes e_{1}+e_{3} \otimes e_{4}+e_{4} \otimes e_{3} \\
& +1 / 2\left(e_{11} \otimes e_{22}+e_{22} \otimes e_{11}+i e_{12} \otimes e_{21}-i e_{21} \otimes e_{12}\right), \\
& \left(\Gamma_{s}\right)_{\Omega}\left(e_{4}\right)=e_{1} \otimes e_{4}+e_{2} \otimes e_{3}+e_{3} \otimes e_{2}+e_{4} \otimes e_{1} \\
& +1 / 2\left(e_{11} \otimes e_{22}+e_{22} \otimes e_{11}-i e_{12} \otimes e_{21}+i e_{21} \otimes e_{12}\right), \\
& \left(\Gamma_{S}\right)_{\Omega}\left(e_{12}\right)=e_{1} \otimes e_{12}-i e_{2} \otimes e_{21}-e_{3} \otimes e_{12}+i e_{4} \otimes e_{21} \\
& +e_{12} \otimes e_{1}+i e_{21} \otimes e_{2}-e_{12} \otimes e_{3}-i e_{21} \otimes e_{4}, \\
& \left(\Gamma_{s}\right)_{\Omega}\left(e_{21}\right)=e_{1} \otimes e_{21}+i e_{2} \otimes e_{12}-e_{3} \otimes e_{21}-i e_{4} \otimes e_{12} \\
& +e_{21} \otimes e_{1}-i e_{12} \otimes e_{2}-e_{21} \otimes e_{3}+i e_{12} \otimes e_{4} .
\end{aligned}
$$

It is therefore the example of Kac and Paljutkin described in ([KP3], 8.19), i.e. the *-algebra $\mathbb{C} \oplus \mathbb{C} \oplus \mathbb{C} \oplus \mathbb{C} \oplus M_{2}(\mathbb{C})$ (with the multiplication and * operation given by the matrix decomposition), the coproduct $\left(\Gamma_{S}\right)_{\Omega}$, the co-unit given by the evaluation in $e_{1}$, and the antipode $\kappa_{s}$.

Since, by construction, the coproducts $\left(\Gamma_{s}\right)$ and $\left(\Gamma_{s}\right)_{\Omega}$ are equal on the subalgebra generated by $(\lambda(1), \lambda(a), \lambda(b), \lambda(a b))$, it is clear that $\lambda(1), \lambda(a), \lambda(b), \lambda(a b)$ are in the intrinsic group of this Kac algebra ([ES2], 1.2.2) (i.e. are group-like elements). Therefore, the dual Kac algebra has at least 4 one-dimensional representations; as it is not abelian (because the Kac algebra we constructed is non-symmetric), it must be equal to $\mathbb{C} \oplus \mathbb{C} \oplus \mathbb{C} \oplus \mathbb{C} \oplus M_{2}(\mathbb{C})$. This Kac algebra is not symmetric (because the initial Kac algebra is not abelian); it is known that the Kac-Paljutkin example is the only possible non-trivial *-Hopf algebra structure on that algebra ([Wi]), and, therefore, the dual Kac algebra is isomorphic to the initial one.

One can easily verify that, if we had taken any 2-cocycle for the function $\omega$, then the deformed coproduct would still had been symmetric, and therefore, it would have been just an isomorphic structure to the initial one.

Of course, it is possible to apply the same ideas to the situation of the group $\mathbb{Z}_{3}>\triangleleft_{\alpha} \mathbb{Z}_{2}$ (where the action of the non trivial element of $\mathbb{Z}_{2}$ is just the permutation 
of the non trivial elements of $\mathbb{Z}_{3}$ ). Then, one can verify that there is no way to obtain a non-symmetric coproduct using the same techniques. In fact, such a Kac algebra, with the same arguments as above, should have at least 3 group-like elements, and, the dual Kac algebra should have $\mathbb{C} \oplus \mathbb{C} \oplus \mathbb{C}$ as a summand, which, as it is dimension 6, implies that it should be abelian; so the initial Kac algebra is necessary symmetric. Anyway, it is known ([Wi]) that there is no non-trivial $*$-Hopf algebra of dimension 6 .

6.5. A Non-Trivial Kac Algebra of Dimension 12. We shall apply now the ideas of 6.4 to construct a non-trivial example of a Kac algebra of dimension 12 .

Let us start with the group $G=\mathbb{Z}_{3} \triangleleft_{\alpha}\left(\mathbb{Z}_{2}\right)^{2}$, where $\alpha$ is the action of $\left(\mathbb{Z}_{2}\right)^{2}=$ $\{1, s, t, s t\}$ on $\mathbb{Z}_{3}=\left\{1, a, a^{2}\right\}$ defined by:

$$
\alpha_{s}(a)=\alpha_{t}(a)=a^{2}, \quad \alpha_{s}\left(a^{2}\right)=\alpha_{t}\left(a^{2}\right)=a .
$$

So, the group $G$ has twelve elements:

$G=\left\{1, a, a^{2}, s, a s=s a^{2}, a^{2} s=s a, t, a t=t a^{2}, a^{2} t=t a, t s=s t, a s t=s t a, t s a^{2}=a^{2} s t\right\}$.

Let us take now $\chi$ a character of $G$; as $a s=s a^{2}$, we get $\chi(a)=1$; therefore, the set of characters of $G$ is, by restriction to $\left(\mathbb{Z}_{2}\right)^{2}$, in bijection with the set of characters on $\left(\mathbb{Z}_{2}\right)^{2}$; so there are 4 different characters on $G$, and as these characters are the dimension 1 subrepresentations of the left regular representation $\lambda$ of $G$, we get, because the group algebra $\mathscr{L}(G)$ is of dimension 12 , that $\mathscr{L}(G)$ is isomorphic to $\mathbb{C} \oplus \mathbb{C} \oplus \mathbb{C} \oplus \mathbb{C} \oplus M_{2}(\mathbb{C}) \oplus M_{2}(\mathbb{C})$. Let $e_{1}, e_{2}, e_{3}, e_{4}, e_{11}, e_{12}, e_{21}, e_{22}, f_{11}, f_{12}$, $f_{21}, f_{22}$ be the matrix units of this algebra; using the above remarks, we can now write the left regular representation $\lambda$ of $G$ as the following:

$$
\begin{aligned}
\lambda(1) & =e_{1}+e_{2}+e_{3}+e_{4}+e_{11}+e_{22}+f_{11}+f_{22} \\
& =e_{1}+p_{1}+e_{2}+p_{2}+e_{3}+q_{1}+e_{4}+q_{2}, \\
\lambda(a) & =e_{1}+e_{2}+e_{3}+e_{4}+j e_{11}+j^{2} e_{22}+j f_{11}+j^{2} f_{22}, \\
\lambda\left(a^{2}\right) & =e_{1}+e_{2}+e_{3}+e_{4}+j^{2} e_{11}+j e_{22}+j^{2} f_{11}+j f_{22}, \\
\lambda(s) & =e_{1}-e_{2}+e_{3}-e_{4}+e_{12}+e_{21}+f_{12}+f_{21} \\
& =e_{1}+p_{1}-e_{2}-p_{2}+e_{3}+q_{1}-e_{4}-q_{2}, \\
\lambda(a s) & =e_{1}-e_{2}+e_{3}-e_{4}+j e_{12}+j^{2} e_{21}+j f_{12}+j^{2} f_{21}, \\
\lambda\left(a^{2} s\right) & =e_{1}-e_{2}+e_{3}-e_{4}+j^{2} e_{12}+j e_{21}+j^{2} f_{12}+j f_{21}, \\
\lambda(t) & =e_{1}-e_{2}-e_{3}+e_{4}+e_{12}+e_{21}-f_{12}-f_{21} \\
& =e_{1}+p_{1}-e_{2}-p_{2}-e_{3}-q_{1}+e_{4}+q_{2}, \\
\lambda(a t) & =e_{1}-e_{2}-e_{3}+e_{4}+j e_{12}+j^{2} e_{21}-j f_{12}-j^{2} f_{21}, \\
\lambda(t a) & =e_{1}-e_{2}-e_{3}+e_{4}+j^{2} e_{12}+j e_{21}-j^{2} f_{12}-j f_{21}, \\
\lambda(s t) & =e_{1}+e_{2}-e_{3}-e_{4}+e_{11}+e_{22}-f_{11}-f_{22} \\
& =e_{1}+p_{1}+e_{2}+p_{2}-e_{3}-q_{1}-e_{4}-q_{2},
\end{aligned}
$$




$$
\begin{aligned}
\lambda(\text { ast }) & =e_{1}+e_{2}-e_{3}-e_{4}+j e_{11}+j^{2} e_{22}-j f_{11}-j^{2} f_{22}, \\
\lambda\left(a^{2} s t\right) & =e_{1}+e_{2}-e_{3}-e_{4}+j^{2} e_{11}+j e_{22}-j^{2} f_{11}-j f_{22},
\end{aligned}
$$

where $j=e^{2 i \pi / 3}$, and $p_{1}, p_{2}, q_{1}, q_{2}$ are the orthogonal projectors defined by:

$$
\begin{aligned}
& p_{1}=1 / 2\left(e_{11}+e_{12}+e_{21}+e_{22}\right), \\
& p_{2}=1 / 2\left(e_{11}-e_{12}-e_{21}+e_{22}\right), \\
& q_{1}=1 / 2\left(f_{11}+f_{12}+f_{21}+f_{22}\right), \\
& q_{2}=1 / 2\left(f_{11}-f_{12}-f_{21}+f_{22}\right) .
\end{aligned}
$$

There exists a standard structure of a symmetric Kac algebra on $\mathscr{L}(G)$ with a symmetric coproduct $\Gamma_{s}$, involution $*$ and a co-involution $\kappa_{s}$ defined, for all $g$ in $G$ by

$$
\Gamma_{s}(\lambda(g))=\lambda(g) \otimes \lambda(g), \quad \kappa_{s}(\lambda(g))=\lambda(g), \quad\left(\lambda_{s}(g)\right)^{*}=\lambda\left(g^{-1}\right) .
$$

One can see that $e_{1}$ is the projection given by the co-unit of $\mathscr{L}(G)$ ([ES2], 6.3.5). The abelian subalgebra generated by $\lambda(1), \lambda(s), \lambda(t), \lambda(t s)$, is generated also by $e_{1}+p_{1}, e_{2}+p_{2}, e_{3}+q_{1}, e_{4}+q_{2}$; it is isomorphic to $\mathscr{L}\left(\left(\mathbb{Z}_{2}\right)^{2}\right)$, and, therefore, by the Fourier transform, to the algebra of functions on $\widehat{\left(\mathbb{Z}_{2}\right)^{2}}$, and this isomorphism sends $e_{1}+p_{1}$ on $\delta_{1}, e_{2}+p_{2}$ on $\delta_{s t}, e_{3}+q_{1}$ on $\delta_{t}$ and $e_{4}+q_{2}$ on $\delta_{s}$ (where $\delta_{x}$ means the Dirac function on $x$, for any $x$ in $\left(\widehat{\left.\mathbb{Z}_{2}\right)^{2}}\right)$. $\widehat{\left(\mathbb{Z}_{2}\right)^{2}}$ :

Let us consider now the function $\omega$ on $\left(\widehat{\left.\mathbb{Z}_{2}\right)^{2}} \times\left(\widehat{\left.\mathbb{Z}_{2}\right)^{2}}\right.\right.$ such that, for all $u, h$ in

$$
\omega(1, u)=\omega(u, u)=1, \overline{\omega(h, u)}=\omega(u, h)
$$

and $\omega(s, t)=\omega(t, s t)=\omega(s t, s)=i$. One can verify that this function is a 2-cocycle on $\left(\widehat{\left.\mathbb{Z}_{2}\right)^{2}} \times\left(\widehat{\left.\mathbb{Z}_{2}\right)^{2}}\right.\right.$. Let $\Omega$ be the unitary element of $\mathscr{L}\left(\left(\mathbb{Z}_{2}\right)^{2}\right) \otimes \mathscr{L}\left(\left(\mathbb{Z}_{2}\right)^{2}\right)$ obtained by the Fourier transform. We get

$$
\begin{aligned}
\Omega= & \left(e_{1}+p_{1}\right) \otimes I+\left(e_{3}+q_{1}\right) \otimes\left[e_{1}+p_{1}+e_{3}+q_{1}+i\left(e_{2}+p_{2}-e_{4}-q_{2}\right)\right] \\
& +\left(e_{2}+p_{2}\right) \otimes\left[e_{1}+p_{1}+e_{2}+p_{2}+i\left(e_{4}+q_{2}-e_{3}-q_{1}\right)\right] \\
& +\left(e_{4}+q_{2}\right) \otimes\left[e_{1}+p_{1}+e_{4}+q_{2}+i\left(e_{3}+q_{1}-e_{2}-p_{2}\right)\right] .
\end{aligned}
$$

We can write $\Omega=\Omega_{1}+i \Omega_{2}$, where

$$
\begin{aligned}
\Omega_{1}= & \left(e_{1}+p_{1}\right) \otimes I+\left(e_{3}+q_{1}\right) \otimes\left(e_{1}+p_{1}+e_{3}+q_{1}\right) \\
& +\left(e_{2}+p_{2}\right) \otimes\left(e_{1}+p_{1}+e_{2}+p_{2}\right)+\left(e_{4}+q_{2}\right) \otimes\left(e_{1}+p_{1}+e_{4}+q_{2}\right), \\
\Omega_{2}= & \left(e_{3}+q_{1}\right) \otimes\left(e_{2}+p_{2}-e_{4}-q_{2}\right)+\left(e_{2}+p_{2}\right) \otimes\left(e_{4}+q_{2}-e_{3}-q_{1}\right) \\
& +\left(e_{4}+q_{2}\right) \otimes\left(e_{3}+q_{1}-e_{2}-p_{2}\right) .
\end{aligned}
$$

As $\omega$ is a 2 -cocycle on $\left(\widehat{\left.\mathbb{Z}_{2}\right)^{2}}\right.$, then $\Omega$ is a 2-cocycle for $\left(\mathscr{L}(G), \Gamma_{s}\right)$. So, $\left(\mathscr{L}(G),\left(\Gamma_{S}\right)_{\Omega}\right)$ is a Hopf-von Neumann algebra; as $\omega$ is involutive, we get that $\left(L(G),\left(\Gamma_{s}\right)_{\Omega}, \kappa_{s}\right)$ is a co-involutive Hopf-von Neumann algebra. 
Moreover, we easily get that $\Omega\left(e_{1} \otimes x\right)=\left(e_{1} \otimes x\right) \Omega=e_{1} \otimes x$, for all $x$ in $\mathscr{L}(G)$; from which we infer that $e_{1}$ still gives a co-unit for the new structure; we easily get:

$$
\begin{aligned}
\Gamma_{s}\left(e_{1}\right)= & e_{1} \otimes e_{1}+e_{2} \otimes e_{2}+e_{3} \otimes e_{3}+e_{4} \otimes e_{4} \\
& +1 / 2\left(e_{11} \otimes e_{22}+e_{22} \otimes e_{11}+e_{12} \otimes e_{21}+e_{21} \otimes e_{12}\right) \\
& +1 / 2\left(f_{11} \otimes f_{22}+f_{22} \otimes f_{11}+f_{12} \otimes f_{21}+f_{21} \otimes f_{12}\right),
\end{aligned}
$$

from which we infer that $\left(\Gamma_{s}\right)_{\Omega}\left(e_{1}\right)=\Gamma_{s}\left(e_{1}\right)$, and, by ([ES2], 6.3.5), we get that this new structure is a Kac algebra.

To prove that this new Kac algebra is non symmetric, it is enough to prove that $\varsigma\left(\Gamma_{s}\right)_{\Omega}(\lambda(a)) \neq\left(\Gamma_{s}\right)_{\Omega}(\lambda(a))$, which is:

$$
\varsigma\left(\Omega_{1}+i \Omega_{2}\right)(\lambda(a) \otimes \lambda(a))\left(\Omega_{1}+i \Omega_{2}\right)^{*} \neq\left(\Omega_{1}+i \Omega_{2}\right)(\lambda(a) \otimes \lambda(a))\left(\Omega_{1}+i \Omega_{2}\right)^{*} .
$$

Let us remark now that $\varsigma \Omega_{1}=\Omega_{1}{ }^{*}=\Omega_{1}, \varsigma \Omega_{2}=-\Omega_{2}{ }^{*}=-\Omega_{2}$. Using these relations, we see that this inequality is equivalent to:

$$
\Omega_{1}(\lambda(a) \otimes \lambda(a)) \Omega_{2} \neq \Omega_{2}(\lambda(a) \otimes \lambda(a)) \Omega_{1},
$$

and it is even enough to prove that:

$$
(p \otimes q) \Omega_{1}(\lambda(a) \otimes \lambda(a)) \Omega_{2} \neq(p \otimes q) \Omega_{2}(\lambda(a) \otimes \lambda(a)) \Omega_{1},
$$

where $p=p_{1}+p_{2}, q=q_{1}+q_{2}$ are in the center of $\mathscr{L}(G)$; this last inequality is easily obtained, using the above expressions for $\Omega_{1}, \Omega_{2}$ and $\lambda(a)$, by direct calculations. Therefore, the Kac algebra in consideration is non-symmetric and we obtain a non-trivial Kac algebra of dimension 12 (i.e. a semi-simple $*$-Hopf algebra of dimension 12 ([ES2], 6.6.9)) which is the $*$-algebra $\mathbb{C} \oplus \mathbb{C} \oplus \mathbb{C} \oplus \mathbb{C} \oplus M_{2}(\mathbb{C}) \oplus$ $M_{2}(\mathbb{C})$ (with the multiplication and $*$ operation given by the matrix decomposition), the coproduct $\left(\Gamma_{s}\right)_{\Omega}$, the co-unit given by the evaluation in $e_{1}$, and the antipode $\kappa_{s}$.

Since, by construction, the coproducts $\left(\Gamma_{s}\right)$ and $\left(\Gamma_{s}\right)_{\Omega}$ are equal on the subalgebra generated by $(\lambda(1), \lambda(s), \lambda(t), \lambda(t s))$, it is clear that $\lambda(1), \lambda(s), \lambda(t), \lambda(t s)$ are in the intrinsic group of this Kac algebra ([ES2], 1.2.2) (i.e. are group-like elements). Therefore, the dual Kac algebra has at least 4 one-dimensional representations.

More precisely, as the number of one-dimensional representations has to divide the dimension of the algebra ([L'R'], 1.6.c), it is easy to see that there are exactly 4 one-dimensional representations, and that the dual Kac algebra must be also equal to $\mathbb{C} \oplus \mathbb{C} \oplus \mathbb{C} \oplus \mathbb{C} \oplus M_{2}(\mathbb{C}) \oplus M_{2}(\mathbb{C})$.

\section{References}

[BS] Baaj, S., Skandalis, G.: Unitaires multiplicatifs et dualité pour les produits croisés de $\mathbb{C}^{*}$ algèbres. Ann. Sci. ENS 26, 425-488 (1993)

[D] Drinfeld, V.G.: Quantum groups. Proceedings ICM Berkeley (1986), vol. 1, pp. 798-820

[ER] Effros, E.G., Ruan, Z.-J.: Discrete quantum groups, I; the Haar measure. Int. J. Math. 5, 681-723 (1994)

[ES1] Enock, M., Schwartz, J.-M.: Algèbres de Kac moyennables. Pacific J. Math. 125, 363-379 (1986)

[ES2] Enock, M., Schwartz, J.-M.: Kac algebras and duality of locally compact groups. Berlin: Springer, 1992

[EV] Enock, M., Vallin, J.-M.: $\mathbb{C}^{*}$-algèbres de Kac et algèbres de Kac. Proc. London Math. Soc. 66, 619-650 (1993) 
[KP1] Кац, Г.И., Палюткин, В.Г. (G.I. Kac, V.G. Paljutkin): Пример кольцевой группы, порожденной группами Ли. Үкр. Матем. Ж. 16, 99-105 (1964)

[KP2] Кац, Г.И., Палюткин, В.Г. (G.I. Kac, V.G. Paljutkin): Пример кольцевой группы восьмого порядка. Үспехи Мат. Наук, 20-5, 268-269 (1965)

[KP3] Кац, Г.И., Палюткин, В.Г. (G.I. Kac, V.G. Paljutkin): Конечные кольцевые группы. Труды Моск. Матем. об-ва 15, 224-261 (1966); Translated in: Finite ring-groups. Trans. Moscow Math. Soc. 251-294 (1966)

[L] Landstad, M.B.: Quantization arising from abelian subgroups. Int. J. Math. 5, 897-936 (1994)

[LR] Landstad, M.B., Raeburn, I.R.: Twisted dual-group algebras: Equivariant deformations of $C_{0}(G)$. J. Funct. Anal. 132, 43-85 (1995)

[L'R'] Larson, R.G., Radford, D.E.: Semisimple Hopf algebras. J. Alg. 171, 5-35 (1995)

[MN] Masuda, T., Nakagami, Y.: A von Neumann Algebra framework for the duality of the quantum groups. Publ. RIMS Kyoto, 30, 799-850 (1994)

[PW] Podleś, P., Woronowicz, S.L.: Quantum Deformation of Lorentz Group. Commun. Math. Phys. 130, 381-431 (1990)

[R1] Rieffel, M.A.: Compact Quantum groups associated with Toral subgroups. Contemp. Math. 145, 465-491 (1993)

[R2] Rieffel, M.A.: Deformation Quantization for Actions of $\mathbb{R}^{d}$. Memoirs A.M.S., 506 (1993)

[TT] Takesaki, M., Tatsuuma, N.: Duality and subgroups. Ann. Math. 93, 344-364 (1971)

[V] Van Daele, A.: Quantum deformations of the Heisenberg group. Proc. Satellite Conference of ICM-90, Singapore: World Sci. 1991, pp. 314-325

[Wa] Wang, S.: Deformation of Compact Quantum Groups via Rieffel's Quantization, to appear in Commun. Math. Phys.

[Wi] Williams, R.: Finite dimensional Hopf algebras. Thesis, Florida State University, 1988

[W1] Woronowicz, S.L.: Tannaka-Krein duality for compact matrix pseudogroups. Twisted $S U(N)$ group. Invent. Math. 93, 35-76 (1988)

[W2] Woronowicz, S.L.: Compact quantum groups. Preprint 1993

Communicated by A. Connes 
